\title{
Extracellular vesicle formation mediated by local phosphatidylserine exposure promotes efficient cell extrusion
}

\author{
Akihito Kira \\ Kyoto Sangyo University \\ Ichiko Tatsutomi \\ Kyoto Sangyo University \\ Keisuke Saito \\ Kyoto Sangyo University \\ Machiko Murata \\ Kyoto Sangyo University \\ Izumi Hattori \\ Kyoto Sangyo University \\ Haruna Kajita \\ Kyoto Sangyo University \\ Naoko Muraki \\ Kyoto Sangyo University

\section{Yukako Oda} \\ Institute for Frontier Life and Medical Sciences, Kyoto University
}

\section{Saya Satoh}

Institute of Cardiovascular Immunology, University of Bonn

\section{Yuta Tsukamoto}

Institute of Cardiovascular Immunology, University of Bonn

\section{Seisuke Kimura}

Kyoto Sangyo University

\section{Hiroki Kato}

Institute of Cardiovascular Immunology, University of Bonn

Tsuyoshi Hirashima ( $\sim$ hirashima.tsuyoshi.2m@kyoto-u.ac.jp)

The Hakubi Center/Graduate School of Biostudies, Kyoto University andJapan Science and Technology Agency, PRESTO https://orcid.org/0000-0001-7323-9627

Kohki Kawane ( $\square$ kawane@cc.kyoto-su.ac.jp )

Kyoto Sangyo University https://orcid.org/0000-0003-1125-9483 
Keywords: Cell Extrusion, Cell Death, Delamination, Extracellular Vesicle (EV), Phosphatidylserine (PS), Epithelium

Posted Date: March 3rd, 2022

DOI: https://doi.org/10.21203/rs.3.rs-257262/v2

License: (c) (1) This work is licensed under a Creative Commons Attribution 4.0 International License. Read Full License 


\section{Extracellular vesicle formation mediated by local phosphatidylserine exposure drives efficient cell extrusion}

Akihito Kira ${ }^{1}$, Ichiko Tatsutomi ${ }^{1 \dagger}$, Keisuke Saito ${ }^{1 \dagger}$, Machiko Murata ${ }^{1 \dagger}$, Izumi Hattori ${ }^{1}$, Haruna Kajita $^{1}$, Naoko Muraki ${ }^{1}$, Yukako Oda ${ }^{2}$, Saya Satoh ${ }^{3}$, Yuta Tsukamoto ${ }^{3}$, Seisuke Kimura ${ }^{4,5}$,

5 Hiroki Kato ${ }^{3}$, Tsuyoshi Hirashima ${ }^{6,7^{*}}$, and Kohki Kawane ${ }^{1^{*}}$

${ }^{1}$ Department of Frontier Life Sciences, Faculty of Life Science, Kyoto Sangyo University, Kyoto, Japan.

${ }^{2}$ Department of Biosystems Science, Institute for Frontier Life and Medical Sciences, Kyoto University, Kyoto, Japan.

$10{ }^{3}$ Institute of Cardiovascular Immunology, University Hospital Bonn, University of Bonn, Bonn, Germany

${ }^{4}$ Department of Industrial Life Sciences, Faculty of Life Science, Kyoto Sangyo University, Kyoto, Japan.

${ }^{5}$ Center for Plant Sciences, Kyoto Sangyo University, Kyoto, Japan.

$15{ }^{6}$ The Hakubi Center/Graduate School of Biostudies, Kyoto University, Kyoto, Japan.

${ }^{7}$ Japan Science and Technology Agency, PRESTO, Kawaguchi, Japan.

*Correspondence: hirashima.tsuyoshi.2m@kyoto-u.ac.jp (T.H.), kawane@,cc.kyoto-su.ac.jp (K.K.)

$20 \uparrow$ These authors contributed equally to this work. 


\section{SUMMARY:}

Cell extrusion is a universal mode of cell removal from tissues, and it plays an important role in regulating cell numbers and eliminating unwanted cells, such as apoptotic, unfit, or cancerous. During this process, cells delaminate from the cell layer, however, the underlying mechanisms remain to be elucidated. Here, we report a conserved execution mechanism of cell extrusion. We found extracellular vesicle (EV) formation in extruding cells at a site opposite to the extrusion direction. Particularly, we found that a lipid-scramblase-mediated local exposure of phosphatidylserine is responsible for EV formation and is crucial for executing cell extrusion, while inhibition of this process disrupted prompt cell delamination and tissue homeostasis.

10 Furthermore, we revealed that the EV formation is governed by the mechanism in microvesicle formation, while the EVs have some hallmarks of apoptotic body. Finally, we illustrated the role of EV formation as promoting the neighboring cells' invasion resulting in the execution of cell extrusion by experimental and mathematical modeling analysis. Taken together, this study provides the insights that membrane dynamics plays a crucial role behind the cell exit from the 15 tissue by connecting the actions of extruding cell and the neighboring cells.

\section{Keywords:}

Cell Extrusion, Cell Death, Delamination, Extracellular Vesicle (EV), Phosphatidylserine (PS), 20 Epithelium 


\section{Introduction}

Numerous unwanted cells are removed from epithelial tissues - in which cells are tightly connected to one another-without disturbing tissue integrity and homeostasis. Cell extrusion is a unique mode of cell removal from tissues, and it is essential to regulate cell numbers and eliminate unwanted cells, such as apoptotic cells, cancer cells, and cells with a lower fitness in cell competition (Gudipaty and Rosenblatt, 2017; Ohsawa et al., 2018; Tanimura and Fujita, 2020). In this process, cells delaminate from the cell layer, to which they initially used to adhere, by the interplay between cell adhesion and cytoskeletal remodeling in both extruding cell and the neighboring cells with their communications (Eisenhoffer et al., 2012; Marinari et al., 2012; Lubkov and Bar-Sagi, 2014; Thomas et al., 2020; Duszyc et al., 2021; Dwivedi et al., 2021). For such communications, S1P produced by extruding cells or mechanotransduction via E-cadherin can drive the reaction of the neighboring cells, which is mainly rearrangement of actomyosin complexes to squeeze out the cells to be extruded. Defects in cell extrusion are considered associated with inflammation and cancer in epithelium (Günther et al., 2012). However, the correlation between them has not yet been evaluated since data on the mechanisms underlying cell extrusion remain limited. In particular, the process whereby the cell exit from the tissue remains a fundamental question which has not been fully addressed. Although various types of actomyosin network (such as apical ring structure, or medial accumulation in extruding cells, and basal radial fiber, apico-basal cable, or supracellular purse-string ring in the neighboring cells) and their contractility has been shown to govern the movement of cell delamination (Kuipers et al., 2014; Michael et al., 2016; Gagliardi et al., 2018; Le et al., 2021; Atieh et al., 2021), whether other mechanisms such as membrane dynamics play a key role in this process remains to be elucidated. 
The dynamics or trafficking of plasma membrane affect cell shape, locomotion, and function in many key cellular processes. Among the membrane trafficking including endocytosis and exocytosis, extracellular vesicle (EV) formation has recently attracted much attention. EVs are classified into mainly three types, namely exosome, microvesicle, and apoptotic body (Akers et al., 2013; Tkach and Théry, 2016; Catalano and O'Driscoll, 2020; Sung et al., 2021). The mechanisms underlying the formation for each EV varies but the exposure of phosphatidylserine (PS) on the outer leaflet of lipid layer, which is also known as "eat me" signal during the engulfment of dying cells by phagocytes, commonly takes place in each EV. Particularly, in microvesicle, PS exposure process is considered required for vesicle formation. EVs are observed during blood coagulation, cell migration, apoptosis, and in various pathological processes. Among them, microvesicle and exosome contain bioactive proteins, nucleic acids, and lipid cargos, which mediate intercellular signal transduction. In contrast, apoptotic body produced in dying cells has been less focused and its physiological role is ambiguous except for the facilitation of being engulfed by fragmenting cell body.

Here, we show that EV formation mediated by local exposure of PS plays a conserved and crucial role in prompting cell exit from the cell layer in various physiological cell extrusion. Experimental and mathematical modeling analyses show that the EV formation contributes to promoting the invasion of the neighboring cells by creating the space, resulting squeezing out the extruding cell. These findings propose a pivotal role of membrane dynamics in cell extrusion and novel EVs function. 


\section{Results}

Local fragmentation of extruding cell and engulfment of the fragments by the neighboring cells

We hypothesized that membrane dynamics play a key role in the execution of cell extrusion. To investigate this, first, we visualized the cell membrane with membrane-bound GFP (membrane GFP) and analyzed the detailed membrane dynamics in both extruding cells and their neighboring cells. In Mardin-Darby canine kidney II (MDCKII) cell monolayers, cell extrusion occurs spontaneously after confluence to adjust cell density (Eisenhoffer et al., 2012). We found extensive budding or blebbing of the cell membrane of extruding cells and the presence of many vesicles inside the neighboring cells via live-imaging analysis of membrane GFP-expressing MDCKII cells (92/92 extrusion events) (Figure 1A; Movie S1). Cleaved caspase-3 is detected via immunostaining in the vesicles of the neighboring cells (110/113 extrusion events) in addition to the cytoplasm of extruding cells (thus most of extruding cells are apoptotic) (Figure 1B). This suggests that these vesicles are derived from extruding apoptotic cells and engulfed by

15 their neighbors.

To verify this, we performed mosaic analysis via the co-culture of RFP-expressing and membrane GFP-expressing cells. The deformation process in extruding cells began with a slight invagination of the apical surface, and after 10-15 min, cells showed budding-like membrane dynamics (Figures 1C, 1D, and S1A; Movie S2). Then, the budding was rapidly fragmented, and the shed fragments were engulfed by the neighboring cells, as shown by the GFP-positive vesicles containing RFP in GFP-expressing cells adjacent to RFP-expressing extruding cells (10/10 extrusion events) (Figure 1D and Movie S2). Consistent with this, the signals for lysotracker staining turned to be positive 30-60 min after the appearance of the vesicles in the 
neighboring cells, indicating that they are phagocytic vesicles and finally fused with the lysosomes (Figure S1B). The fragmentation and engulfment continued for 10-15 min and a remaining portion (obviously larger than each fragment) of the extruding cell after fragmentation was squeezed out from the cell layer to the apical direction (Figures 1C and S1A). Thus, the extruding cells follow two fates: several fragments (smaller size) engulfed or a cell body (larger size) extruded. Interestingly, fragmentation and engulfment occurred preferentially at the lateral site of basal half in the extruding cells (10/10 extrusion events) (Figure 1E). In addition, the protrusion of the extruding cell from the cellular monolayer began simultaneously as fragmentation and engulfment (Figures $1 \mathrm{C}$ and $1 \mathrm{~F}$ ). These results suggest that fragmentation and/or engulfment are involved in the exit from cell layer, thus execution of cell extrusion.

\section{The fragmentation of extruding cell is mediated by phosphatidylserine exposure and plays} a crucial role in the execution of cell extrusion

Apoptotic cells expose PS as an "eat-me" signal and are engulfed by phagocytes (Nagata et al., 2010). Staining results with Annexin V, a PS-binding protein, showed that extruding cells exposed PS, while signals were enriched at the basolateral and basal parts with a patched pattern (Figure 2A). This strongly suggests that the cell fragments expose PS, and are recognized and engulfed by neighboring cells.

To evaluate the function of engulfment during cell extrusion, we intended to disturb the engulfment process using the MFG-E8 mutant protein. Secreted MFG-E8 protein binds to both PS exposed on apoptotic cells and integrins on phagocytes to promote phagocytosis, whereas the MFG-E8-D89E mutant protein (D89E), which carries a mutation in the integrin-binding motif, inhibits PS-mediated phagocytosis in a dominant-negative manner (Hanayama et al., 2002). We 
expected that D89E (mouse)-overexpression in MDCKII cells prevents engulfment after fragmentation, resulting in unengulfed fragments in the intercellular space. However, the fragmentation process, rather than the engulfment, was impaired. Half of the cell extrusion showed delayed fragmentation, thus the budding was not detected even after 100 min or more following the deformation of the cell shape (Figure 2B). These extruding cells with delayed fragmentation, despite the continuous rounding-like membrane deformation, showed impaired protrusion from the cell layer and prolonged time to complete extrusion (dethatching from the cell layer) (Figures 2B and 2C). Furthermore, upon D89E expression, some cleaved caspase-3positive cells were found in the cell layer without protrusion, indicating that the execution of cell extrusion but not apoptosis is impaired (Figure 2D). These results suggest that fragmentation and/or engulfment is critical for prompt cell extrusion.

Another mammalian epithelial cell line, EpH4, showed fragmentation (budding and shedding) during cell extrusion (61/61 extruding cells) at the basal half of extruding cells (7/7 extruding cells) as observed in MDCKII cells. However, the fragments were not rapidly engulfed

15 by neighboring cells but were moved along the basolateral intercellular space to leave away (Figure 2E; Movie S3A). The masking of PS by the D89E mutant in EpH4 cells inhibited fragmentation and remarkably cell extrusion, although the engulfment of the fragments is rarely observed in the cell line (Figures 2F and 2G; Movie S3B). The results from these two cell lines indicated that fragmentation, which is mediated by PS exposure, is a common process and

20 promotes the protrusion of extruding cells. PS exposure in apoptotic cells is governed by the Xkrelated protein (Xkr) lipid scramblase family, particularly Xkr4, Xkr8, and Xkr9 (Suzuki et al., 2013; Suzuki et al., 2014). The knockdown of $X k r 8$, the most abundant among the three in EpH4 cells (Table S1), decreased PS exposure and caused abnormal fragmentation in the budding 
process, impaired protrusion, and longer extrusion (Figures 2H-2J), whereas the knockdown of TMEM16F (Ano6), the most abundant lipid scramblase belonging to the TMEM16 family in EpH4 cells (Table S1), did not affect PS exposure, cell fragmentation, nor extrusion (Figures S2A-S2C).

\section{Universality of the fragmentation of extruding cell and its function for cell extrusion in vivo}

Fragmentation was also observed in cell extrusion of UV-induced apoptotic cells (Figure S3A). To further show that the fragmentation occurs in vivo, time-lapse imaging was performed on the pupal dorsal abdominal epidermis of Drosophila. During the early pupal stage, all large-sized larval epithelial cells (LECs) extrude in the basal direction and then were replaced by small-sized adult epithelial cells (histoblasts) through their frequent cell division and movement (Nakajima et al., 2011; Teng et al., 2017; Michel and Dahmann, 2020; Hoshika et al., 2020) (Figure 3A). Upon extrusion of a lateral membrane-localized GFP (Lgl:GFP)-expressing LEC adjacent to other LECs, fragmentation of extruding cell was observed (23/23 extruding cells) (Figure 3B;

15 Movie S4). In mosaic analysis in which RFP was expressed in LECs only whereas Lgl:GFP was expressed in both LECs and histoblasts (Figure 3C), RFP-positive vesicles derived from extruding LECs were detected in the neighboring histoblasts ( 5 of 5 extrusion events), indicating that fragmentation (and engulfment by the neighboring LECs and histoblasts) occurs during physiological cell extrusion in vivo. Apical fragmentation occurred after the apical surface, which was formerly occupied by extruding cells, was closed and covered by their neighbors (10/10 extrusion events) (Figure 3D). Interestingly, the fragmentation and engulfment were observed preferentially at the apical half of the extruding cells (4/5 extrusion events), which was also in the opposite direction of extrusion. 
The ectopic expression of secreted Annexin V-EGFP fusion proteins (Annexin V:GFP) (Sapar et al., 2018) in histoblasts detected PS exposure in a speckled manner at the apical sites of extruding LECs, which are the likely sites of fragmentation, after the cell started to deform (13/13 extruding cells) (Figure 3E; Movie S5), whereas the expression of the mutant Annexin V:GFP (unable to bind to PS) did not (0/22 extruding cells). This result suggests that spatiotemporally regulated PS exposure triggers fragmentation. Annexin V-expression caused abnormality in the fragmentation (less number and smaller size) (11/16 of Annexin Vexpressing extruding cells vs 0/11 in the case of Annexin V mutant) (Figure 3F) and extrusion most likely owing to masking the exposed PS. The extruding cell stayed longer time in the epithelial cell layer even after the detaching apical extracellular space shown by time lapse analysis and thus many round-shape cells on the way to extrude were observed in the cell layer expressing Annexin V, whereas in the control situations the extruding cells leave the cell layer within 20 min (Figure 3G). Furthermore, when Annexin V was expressed in LECs, the adult dorsal abdomen showed loss of continuity of the epidermis and bristles at the midline (Figure

$153 \mathrm{H}$ ), a severe defect in cell death or extrusion as previously reported (Michel and Dahmann, 2020). The knockdown of the Drosophila Xk gene (Xk or CG32579), a single homolog of the mammalian Xkr family, showed smaller number of fragmentations in extruding cells (12/13 in $X k$-knockdown vs 2/16 in control) (Figure 3I). The progression of cell extrusion after the closure of the apical surface of the extruding cells was impaired in the $X k$-knockdown epidermis as 20 evidenced by the persistence of extruding cells in the cell layer for an extended time (Figure 3J). The replacement of larval cells by histoblasts was also delayed in the $X k$-knockdown pupal epidermis (Figure $3 \mathrm{~K})$.

In another physiological cell extrusion, during cell turnover in the adult intestinal 
epithelium, fragmentation and engulfment occurred in the Drosophila midgut as indicated by the formation of some vesicles in the neighboring cells (Figures S3B and S3C), as well as in the mouse intestinal organoid as shown by time-lapse imaging results (Figures S3D and S3E; Movie S6). When $X k$ was knocked down in an enterocyte-specific manner in the Drosophila midgut, an increased tissue width and shorter lifespan was observed (Figures S4A-S4C). These results indicate that PS exposure-induced fragmentation of the extruding cells is a universal process that plays a significant role in the execution of cell extrusion to ensure tissue development and homeostasis.

\section{The fragmentation of extruding cells is a formation of extracellular vesicles}

We speculated that fragmentation involves the local formation and release of EVs. In EVs, including exosome, microvesicle, and apoptotic body, PS exposure and actomyosin accumulation at the budding sites, mediated by the function of the Arf family and Phospholipase D (PLD), are important for microvesicle formation (Muralidharan-Chari et al., 2009; Schlienger

15 et al., 2014). In addition to the requirement of PS exposure for the fragmentation (Figures 2B, $2 \mathrm{C}, 2 \mathrm{~F}, 2 \mathrm{G}, 2 \mathrm{I}, 2 \mathrm{~J}, 3 \mathrm{~F}$ and $3 \mathrm{I}$ ), actin or myosin accumulated around the root of the budding sites of the fragments in MDCKII cells (10/10 extruding Actin:GFP expressing cells and 33/39 Lifeact:mRuby expressing extruding cells) and Drosophila LECs (5/5 extruding cells) (Figures 4A and 4B). When Arf6, Arfl, or Pldl was knocked down in EpH4 cells, impaired fragmentation

20 and delayed extrusion with defective protrusion were observed (Figures 4C and 4D). Extruding EpH4 cells with Arf6 or Pld1-knockdown showed local PS exposure (control; 77\%, Arf6knockdown; 55\%, and Pld1-knockdown; 58\% of extruding cells). These results are consistent with the fact that the PS exposure precedes EV formation and indicate that PS exposure promote 
cell extrusion via EV formation. Arf51F (homologous to mammalian Arf6) accumulated at the apical but not the basal part of extruding cells in Drosophila pupal epidermis during fragmentation (6/6 extrusion events) (Figure 4E). Arf51F-knockdown in the pupal epidermis showed impaired fragmentation and delayed progression of cell extrusion (Figures 4F and 4G). Furthermore, the knockdown of $\operatorname{Arf79F}$ (homologous to mammalian Arf1), which is more abundant than $A r f 51 F$, in the Drosophila midgut (Table S1) in an enterocyte-specific manner resulted in enlarged midguts and shorter lifespans (Figures S4D-S4F). These data indicate that the fragmentation of the extruding cells is the spatiotemporally restricted EV formation governed by a similar mechanism as microvesicle formation and promotes cell protrusion, thus the execution of cell extrusion.

In parallel, the hallmarks of EVs formed in extruding cells fulfill the criteria of apoptotic body. Although the size of vesicles varies, $40 \%$ of vesicles have more than $3 \mu \mathrm{m}$ diameter (Figure 4H). The formation of EVs including the larger sized vesicles were inhibited by pld or arf family gene knockdown (Figure 4C). In addition, signals for DNA staining or Histone

15 H2B:GFP (data not shown) were positive in substantial part of EVs evaluated in phagocytic vesicles and vesicles in intercellular space (a positive example: Figure 4I and a negative example: Figure 1B). During the formation of EV, fragmented nuclei are evident in the bud (Figure 4I). As the EVs appeared to show hallmarks of both microvesicle and apoptotic body, we speculated some mechanisms of formation of both EVs are common. To test this, we utilized 20 FDC-P1 cells, a mouse myeloid progenitor cell line, which show obvious apoptotic body formation upon its death (Figure 4J; Movie S7A; 10/10 apoptotic cells). Treatment of PLD-1 inhibitor strongly reduced cell fragmentation, thus apoptotic body formation in IL-3 deprivationinduced apoptosis (Figure 4J and Movie S7B; 1/10 apoptotic cells showed apoptotic body 
formation). This result suggested that apoptotic body formation is governed by a similar mechanism to that of microvesicle formation and that it is an important process for the cell to leave the cell layer.

\section{Extracellular vesicles drive cell extrusion via the invasion of neighboring cells}

We explored the mechanism by which the EVs propel the execution of cell extrusion. First, we examined the possible role of EV formation for the basal invasion of the neighboring cells' lamellipodium, a driving force for cell extrusion (Kocgozlu et al., 2016; Le et al., 2021). When plotting the evolution of the area at the plane of most basal (Figure 5A: Most basal) in MDCKII, we observed a decrease during cell extrusion, however the reduction of cell area had occurred prior to the shedding of vesicles (i.e., EV formation) (Figures 5A and 5B; Movie S8A). Also, during LECs extrusion, EV formation at the apical part occurred following the closure of apical surface by presumably lamellipodial invasion of neighboring cells (Figure 3D), further suggesting that the EV formation does not promote lamellipodial invasion by neighboring cells. Next, we assessed the region where EV formation most frequently occurs, located at basal half but closed to the border of apical and basal half in cultured cells (Figure 5A: BasalMiddle). The area of the extruding cells at the plane decreased during cell extrusion, and was most evident during EV formation in MDCKII cells (Figures 5A and 5B; Movie S8B). The reduction of cell area at the Basal-Middle plane during a 10-minutes EV shedding reached 50\%, whereas approximately $10 \%$ of area reduction was observed at the most basal plane (Figures 5B and 5D). Such prominent area reduction at the plane where EV formation occurs was also observed in mouse intestinal organoids and Drosophila LECs (in the case of LECs, just below the most apical plane, with the apico-basal axis being opposite) (Figures 5D and S3F-S3H). 
Furthermore, when the EV formation was delayed by the expression of MFG-E8 D89E mutant in MDCKII cells or expression of AnnexinV in Drosophila pupal epidermis, the prominent reduction of the area in extruding cells coincided with EV formation (the area was not largely reduced until the EV formation) and delayed in accordance with the delayed EV formation (Figures 5C and 5D). Particularly, in Annexin V-expressing pupal epidermis, extruding LECs stopped both EV formation and area reduction at the Basal-Middle plane after the 10-15-minutes EV formation, followed by staying longer time in the epithelial cell layer (6/6 extruding cells) (Figure $3 \mathrm{G}$ and data not shown). These results strongly suggested that the area reduction is caused by EV formation and it is crucial for the cells to exit from the cell layer. by the extruding cell, with actin accumulation at the leading edge (Figures 5A and 5E). Putting it all together, these data showed that EV formation plays a role in space formation for neighboring cells' invasion to promote the execution of cell extrusion at the basal portion, but not most basal plane, via a subcellular space competition (Figure 5F).

\section{EV-mediated space competition promotes cell extrusion}

Finally, we developed a mathematical model to better understand the role of EV formation during cell extrusion. The model represents the dynamics of cells and EVs on the vertical section of the cell monolayer, where the medium and substrate are respectively located at the top and bottom sides of the simulation windows, assuming the condition of cell cultures on a dish (Figures 6A and 6A'). In this section, we refer to the medium side of the cells as the 'apical side', and the substrate side as the 'basal side'. In simulations under the standard setting, the center of a virtual cell monolayer was specified as an extruding cell at a given time, and cell-cell 
adhesion between the extruding cell and its neighboring non-extruding cells decreased during the extrusion process (Denning et al., 2012; Grieve and Rabouille, 2014; Teng et al., 2017). The neighboring non-extruding cells then spread their lamellipodia at the basal side, consistent with our results (Figure 5A; Movie S8A) as well as previous reports (Kocgozlu et al., 2016; Le et al., 2021). Immediately after the lamellipodia protrusions, the extruding cells began generating EVs on the cell periphery with diameters similar to our observations of their turnover (Figure S5A).

First, we confirmed whether the mathematical model could explain the observed cell extrusion dynamics. The model simulations were performed with the parameter set referring to experimental data (Figures S5B-S5E), and they successfully reproduced the spatiotemporal dynamics of cell extrusion, which fit well with the experimental data (Figures 6B and 6C; Movie 9). In contrast, without EV formation, the length of extrusion increased less over time, even though the neighboring non-extruding cells invaded the most basal region in contact with the substrate due to the lamellipodia protrusions (Figures 6B and 6C; Movie 9). This indicated that EV formation promoted cell extrusion. location of EV formation. To this end, we considered three virtual cases where EV formation occurs on different sides according to cell polarity, including the basal, lateral, and apical sides. In the case of basal EV formation, both the apical and basal edges of the extruding cell moved to the medium side, and cell extrusion was evident (Figure 6D, upper panel). In the case of lateral EV formation, the apical cell edge, but not the basal cell edge, moved to the medium side (Figure $6 \mathrm{D}$, middle panel). These results suggest that neighboring non-extruding cells occur in the region where the EVs were formed. As anticipated, cell extrusion did not occur in the case of apical EV formation (Figure 6D, lower panel), showing that EV formation would determine the orientation 
of cell extrusion.

Finally, we further explored the impact of other model parameters on cell extrusion, such as the EV turnover rate $(\gamma)$ and cell-cell adhesion of the extruding cell $(\Delta)$. Interestingly, the length of extrusion was maximized at intermediate EV turnover rate values, regardless of the changes in cell adhesion (Figure 6E). When EV turnover was too slow $\left(\gamma=10^{-4}\right)$, EVs remained in the region where they are formed; thus, the neighboring non-extruding cells had a lower chance to enter the space (Figure 6Fa; Movie S10). In contrast, when EV turnover was too fast $\left(\gamma=10^{0}\right)$, the neighboring non-extruding cells did not have sufficient time for appropriate deformation to eliminate the extruding cell (Figure 6Fb; Movie S10). These results suggest that EV turnover generated by extruding cells regulates space competition with neighboring non-extruding cells at the subcellular scale. Moreover, our simulation showed that the decrease in cell-cell adhesion of the extruding cell promoted cell extrusion (Figure 6Fc; Movie S10), which is consistent with the conclusions of previous studies (Denning et al., 2012; Grieve and Rabouille, 2014; Teng et al., 2017). In summary, the simulation analysis corroborates that EV formation and turnover, in

15 addition to the decrease in cell-cell adhesion, promote cell extrusion from monolayer tissues.

\section{Discussion}

Our findings reveal that local PS exposure and subsequent EV formation mediated by PLD and the ARF family in extruding cells is a conserved mechanism that promotes efficient cell extrusion. Prolonging this extrusion process caused defects in epithelial tissue, suggesting that the mechanism shown in this study is the core machinery of cell extrusion and that prompts execution of extrusion is critical for tissue homeostasis. We also showed the unexpected function of PS exposure and EV formation in cell extrusion, and suggest that other than the "eat-me" 
signal for the engulfment, there are multiple different roles of PS exposure during cell demise. The known function of EVs is intercellular signal transduction via its cargos after the release of EVs and fusion to target cells (Akers et al., 2013; Tkach and Théry, 2016; Gupta et al., 2017)(Catalano and O'Driscoll, 2020; Sung et al., 2021). However, the formation of the EVs and cell protrusion occurred simultaneously, suggesting a function of EVs which is independent of signal transduction. The EVs derived from extruding cells have both hallmarks of microvesicle and apoptotic body. The formation of the EVs is inhibited by the knockdown of arf or pld family genes related to microvesicle formation, whereas the EVs contains DNA or histone inside and the vesicle size is large enough as apoptotic body. The physiological role of the apoptotic body is believed to be engulfed efficiently by phagocytes or sometimes the significant function is suspicious. Our results suggest not only the significant role of the apoptotic body in the epithelial cellular end but also propose that the apoptotic body is formed by a similar mechanism as the one used in microvesicle formation.

Given that the EV formation at the site opposite to the direction of extrusion in the

15 apico-basal axis concurrently with the cell protrusion to extruding direction, membrane dynamics in extruding cells contributes to producing a driving force of extrusion, in addition to the actomyosin complex formed by the neighboring cells and/or extruding cells (Kuipers et al., 2014; Michael et al., 2016; Gagliardi et al., 2018; Le et al., 2021; Atieh et al., 2021) and it might control extrusion directionality. Also, it highlights the importance of subcellular events at basal parts (in the case of apical extrusion) in polarized epithelia as reported in cell intercalation (Sun et al., 2017).

Both imaging analysis and computational simulation supported the idea that EVs formation drives the execution of cell extrusion by promoting the invasion of neighboring cells 
via a kind of subcellular space competition at the just upper plane of the most basal plane. Lamellipodial protrusion at the most basal plane proceeds to this event, indicating an overall view, in which the many ordered sequential events in both neighboring cells and extruding cells are essential for efficient cell extrusion. Computational simulation points out the importance of the turnover of EVs to make space for space competition. The turnover of EVs is achieved via either the engulfment by neighboring cells or moving of EVs. The formation, shedding, and disappearance of EVs are essential parts, while in some cell competitions the engulfment of whole loser cells is important for its elimination (Li and Baker, 2007; Ohsawa et al., 2011). In the case of elimination of oncogenic cells a vesicle formation removes apical determinant including adherens junction to render the cells basal extrusion (Fadul et al., 2021). The role of the EVs shown in our study is to make space for the neighboring cells' invasion and not related to pinch off the apical adhesion apparatus. The timing of disappearance of adherence junction and EVs formation is different, and the E-cadherin is not detected in the EVs in mammalian cell lines and Drosophila LECs (Figures S6A, S6B, and data not shown). Oncogenic cells might

15 hijack and modify the mechanism underlying the EVs formation used in general cell extrusion for their survival and tumor invasion as cancerous cells sometimes utilize and modify any endogenous machinery such as the machinery for cell division (Anton et al., 2018).

Our findings provide important insight into how cells exit from tissues, a fundamental cell behavior that is also observed in other processes including cancer cell invasion and neural cell differentiation (Ohsawa et al., 2018). Future studies on the mechanism of spatiotemporally restricted PS exposure and EV formation, as well as their interplay with the actomyosin dynamics and cell-cell adhesion dynamics, will provide deeper insights into the execution of cell extrusion. Knockdown of genes that lead to impaired EVs formation and cell extrusion with 
digestive tract specific manner in Drosophila can shorten the lifespan (Figures S4A-S4F). Considering that the impaired cell extrusion leads the disfunction of epithelial barrier (Gu et al., 2015; Duszyc et al., 2021) and that disablement of barrier in digestive tract shorten the lifespan (Resnik-Docampo et al., 2017), EV formation in extruding cells might maintain the homeostasis in the gut to keep its barrier function. Further analysis on cell extrusion in vivo with a view from EVs formation will expand our understanding of the relationship between impaired cell extrusion and epithelial diseases, including cancer and inflammation. 


\section{Acknowledgment}

We thank M. Nakahara, Y. Yanagihashi, and N. Yamada for their contribution in the discussion and experimental support. This work was supported by the Japan Science and Technology Agency [Grant number JPMJPR14M2 to K.K.]; the Japan Society for the Promotion of Science [grant number 26713011 to K.K. and $21 \mathrm{H} 05290$ to T.H.]; the Ministry of Education, Culture, Sports, Science \& Technology of Japan [Grant No. S1511023 to S.K.]; the Deutsche Forschungsgemeinschaft (DFG, German Research Foundation) [Grant No. EXC 2151 390873048 to H.K.]; the Takeda Science Foundation; the Mochida Memorial Foundation for Medical and Pharmaceutical Research; and the Uehara Memorial Foundation to K.K..

\section{Declaration of interests}

Authors declare no competing interests.

\section{Author contributions}

A.K., H.K., T.H., and K.K. designed the experiments and wrote the paper. A.K, M.M., K.S., I.T., I.H., H.K., N.M., S.S., Y.T., S.K., T.H., and K.K performed experiments and analyzed the data. Y.O. and S.K. devised the methodology and edited the manuscript.

\section{Data and materials availability}

RNAseq read data are available in the DDBJ Sequenced Read Archive under the accession numbers DRA011352.

\section{Code availability}


Relevant codes for this research work are stored in GitHub:

https:/github.com/tsuyoshihirashima/EVs-CellExtrusion.git, and will be archived within the Zenodo repository after the acceptance of manuscript. 
Figures

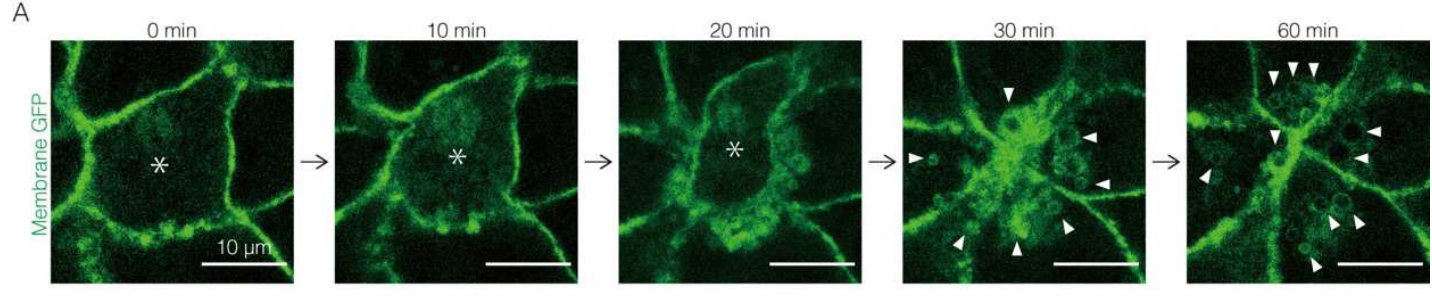

B

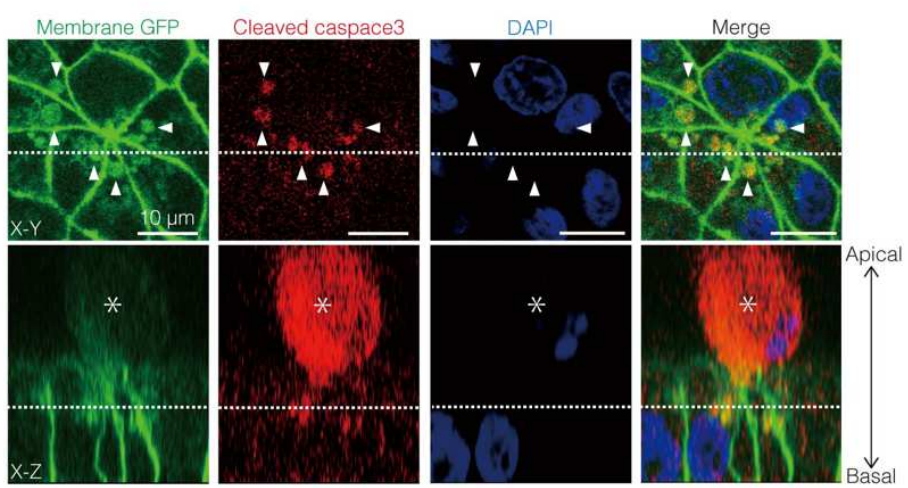

C
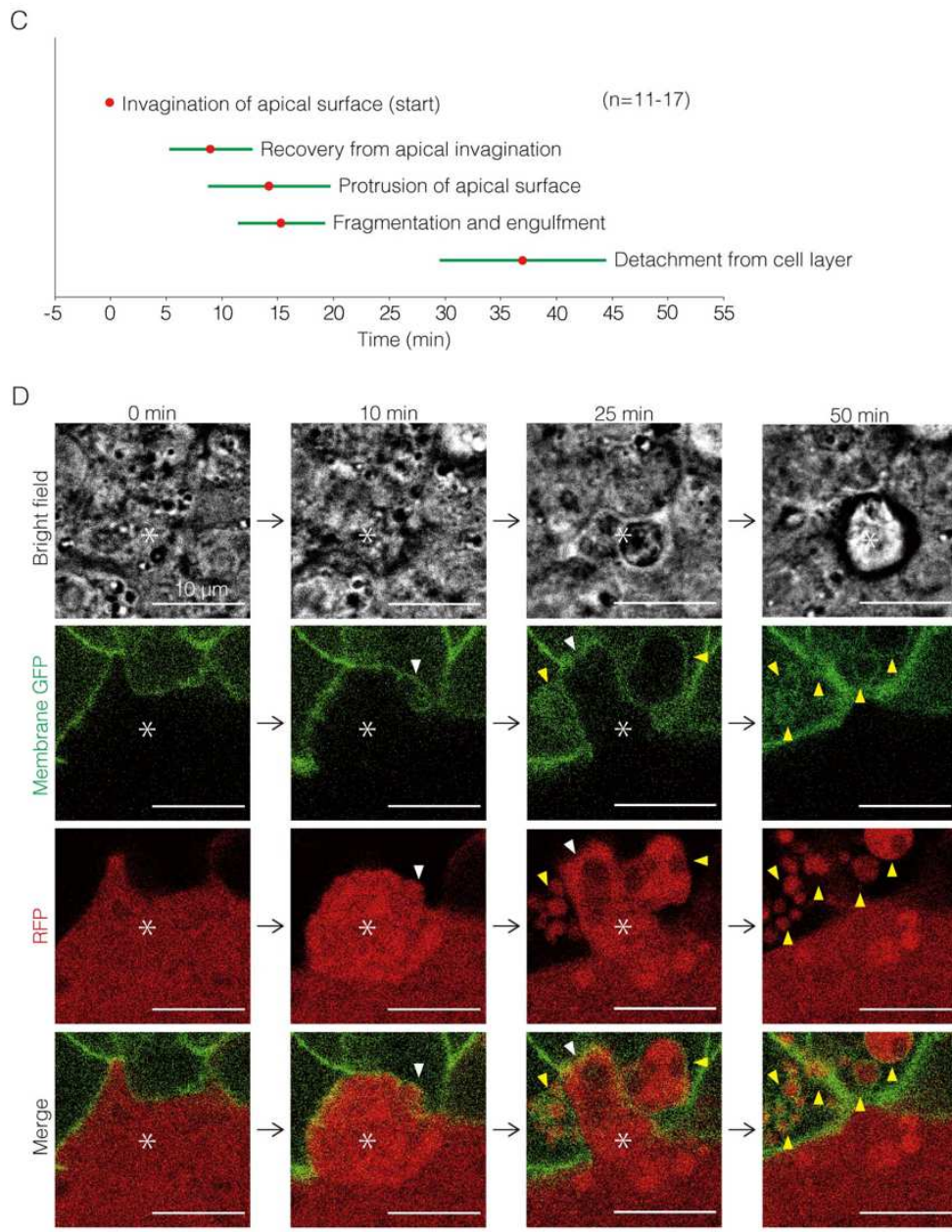

Fig. 1A-D 
E

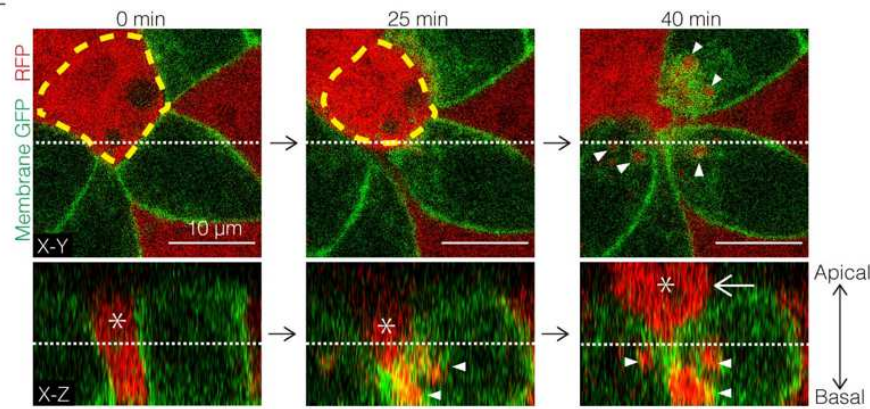

F
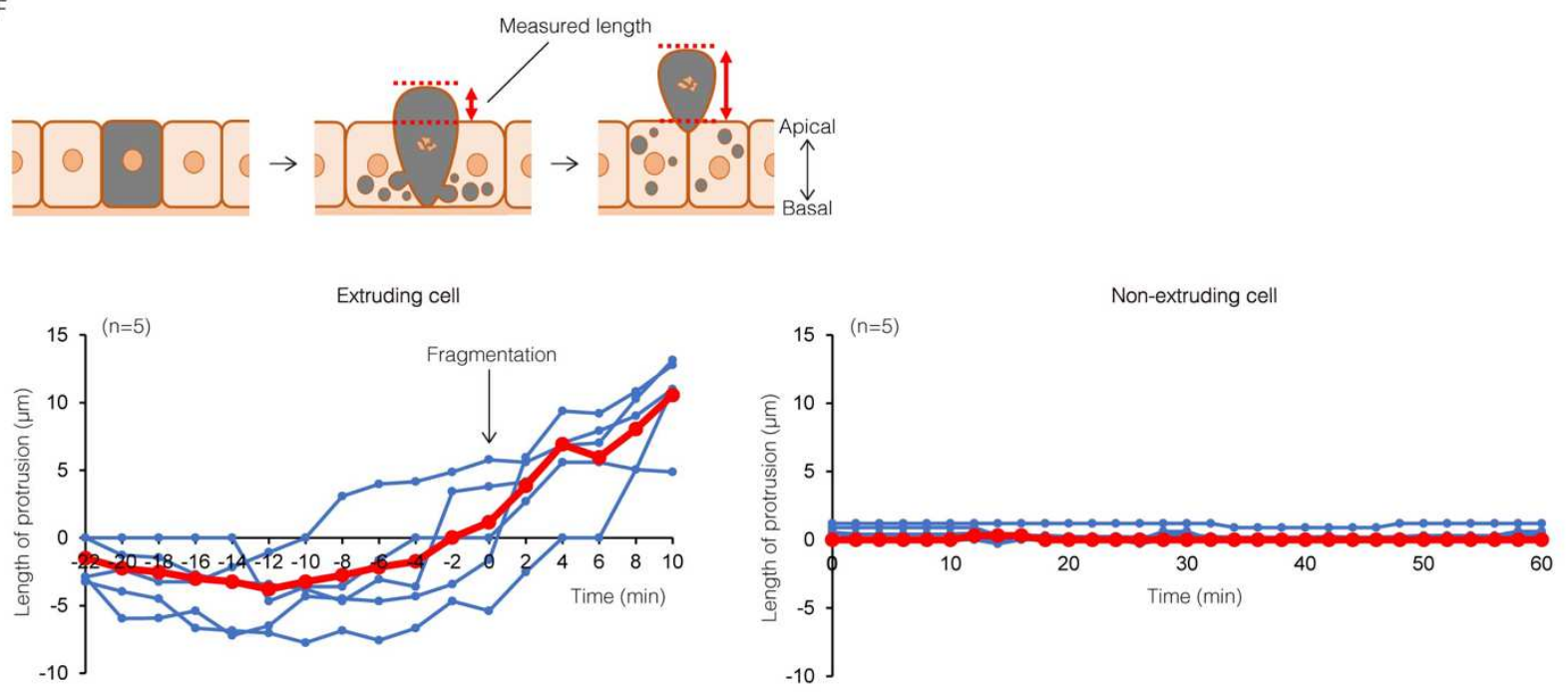

Fig. 1E-F 
Figure 1. Fragmentation of extruding cells and engulfment of the fragments by the neighboring cells in mammalian cultured cells

(A) Vesicles (arrowheads) in the neighboring cells of an extruding cell (asterisks) in palmitoylated GFP (membrane GFP)-expressing MDCKII cells.

(B) Immunostained membrane GFP-expressing MDCKII cells for cleaved caspase-3 with DAPI. Arrowheads indicate vesicles in the neighboring cells of an extruding cell (asterisks) and dashed lines indicate the corresponding position in another angled view (x-y or x-z images).

(C) Schematic illustration of the processes during cell extrusion in MDCKII cells. Average (circles) and standard deviation (lines) of the time after the start of cell extrusion (defined as the invagination of apical surface) are shown. Fragmentation is defined as budding and shedding of vesicles formed in extruding cells.

(D) Co-culture between membrane GFP- and RFP-expressing MDCKII cells. White arrowheads indicate membrane budding in an RFP-expressing extruding cell (asterisks), whereas yellow arrowheads indicate the vesicles containing RFP with GFP-labeled membrane in GFP-expressing 15 neighboring cells.

(E) Fragmentation at the basal part (arrowheads) and concurrent apical protrusion (arrow) in an RFP-expressing extruding cell (asterisks) under co-culture conditions ( $\mathrm{x}-\mathrm{y}$ and $\mathrm{x}-\mathrm{z}$ images).

(F) Quantification of the length between the apical surface of the extruding or non-extruding cell and the apical surface of the cell layer as the diagram shows. Time 0 in extruding cell is defined 20 as the start of fragmentation. Blue lines and Red bold lines indicate length of protrusion in individual extruding cell and mean value, respectively. 

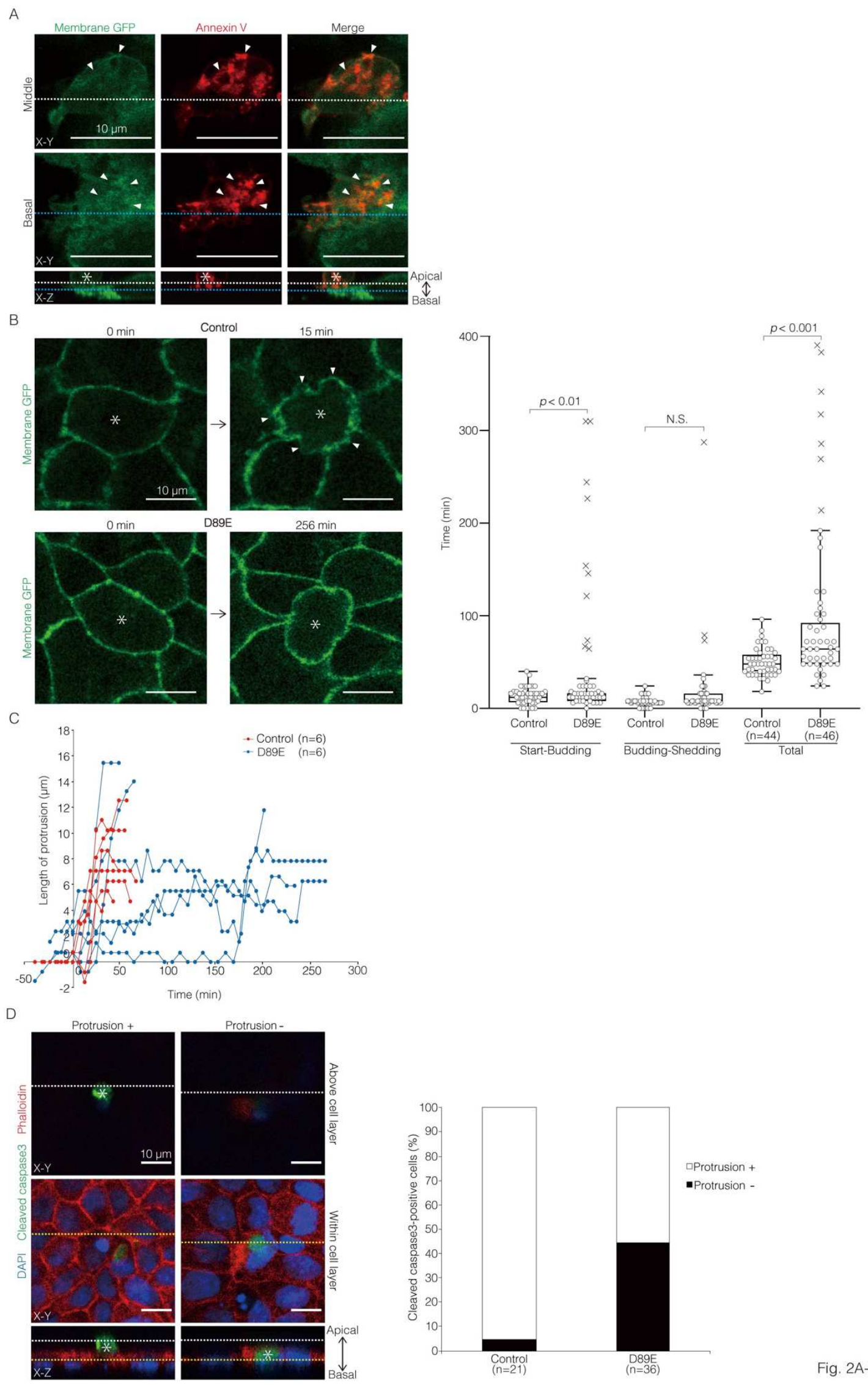

Fig. 2A-D 
E
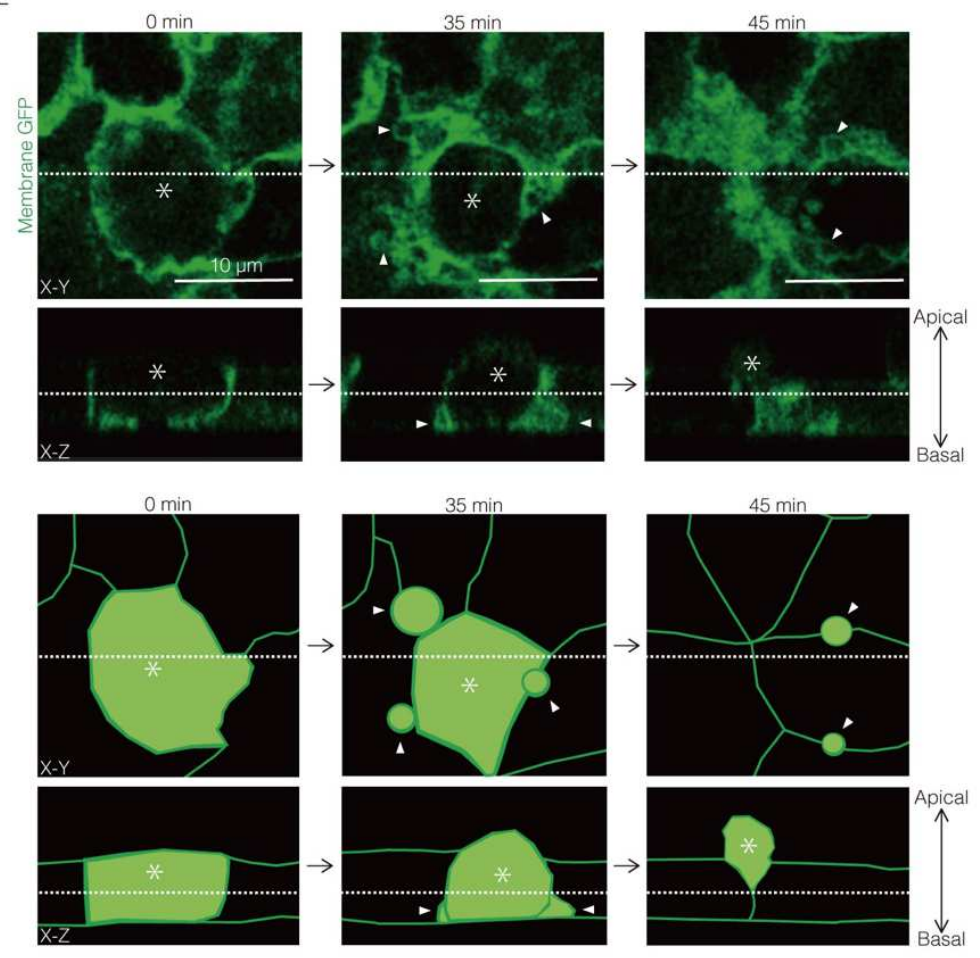

F
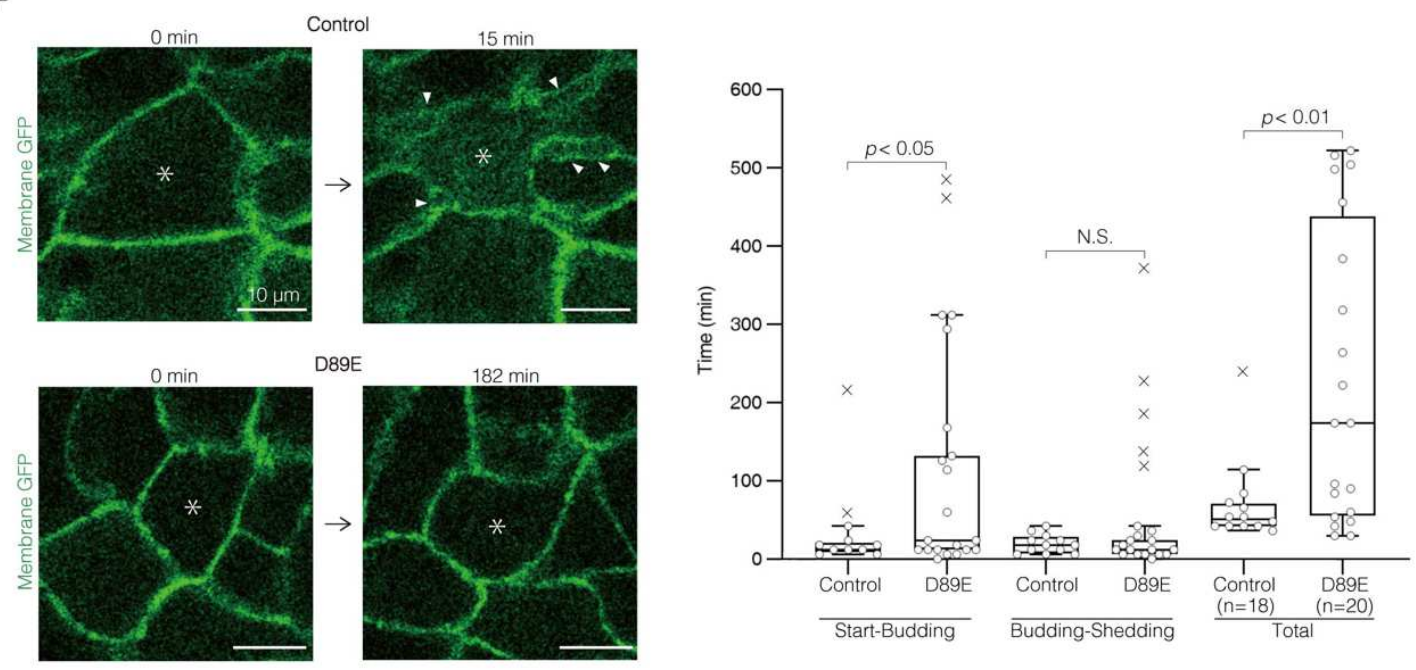

G

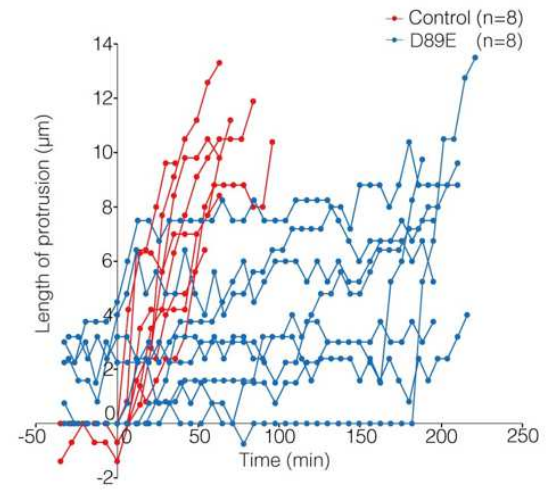

Fig. 2E-G 
$\mathrm{H}$
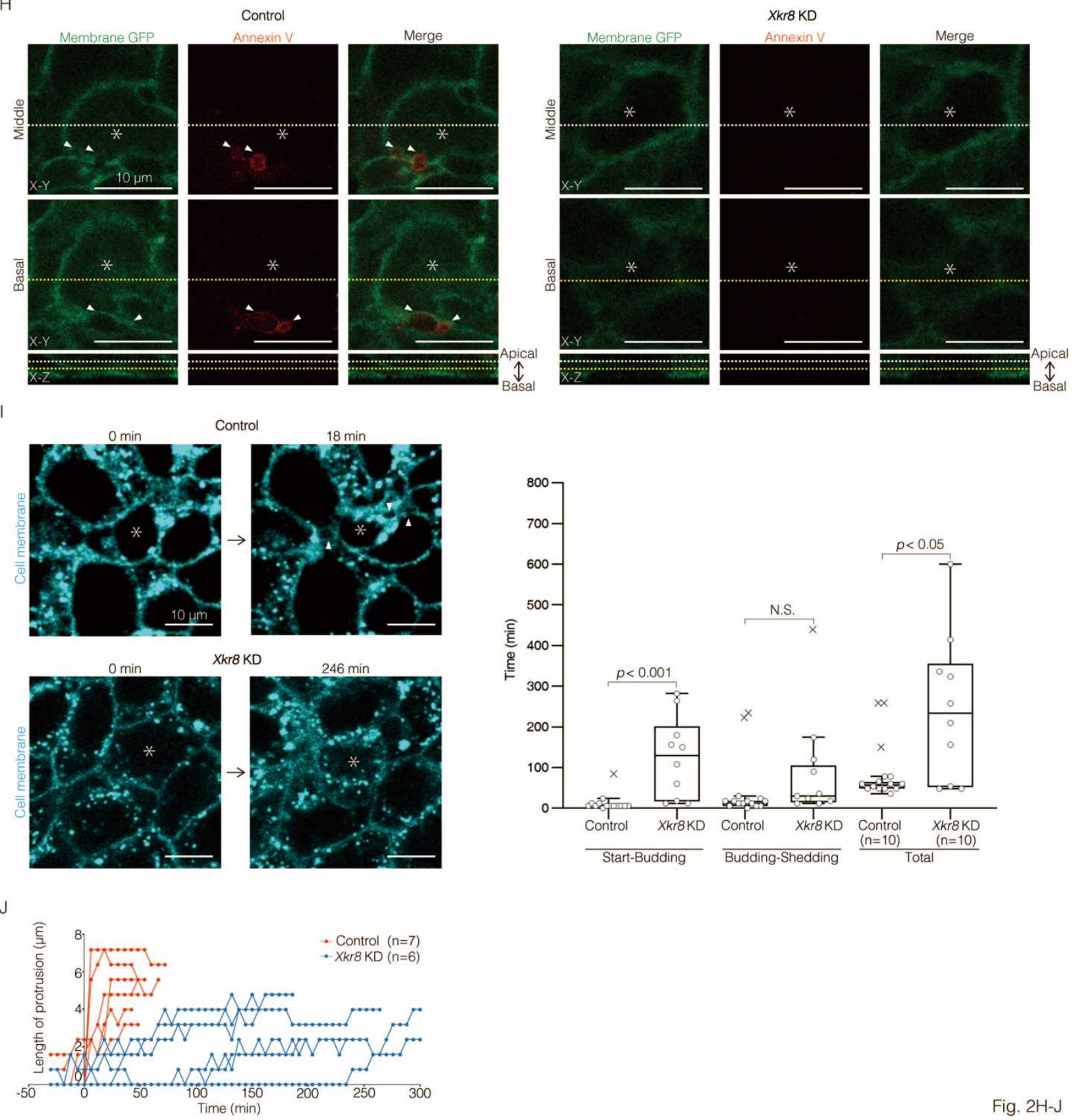

Fig. $2 \mathrm{H}-\mathrm{J}$ 
Figure 2. The PS exposure-mediated fragmentation of extruding cells promotes the execution of cell extrusion in mammalian cultured cells

(A) An Annexin V-stained membrane GFP-expressing extruding MDCKII cell (asterisks) in the middle and basal x-y sections with x-z sections. Not uniform but speckled pattern of stained signals (arrowheads) are shown.

(B) Delayed fragmentation of membrane GFP-expressing extruding MDCKII cells with MFG-E8 D89E-overexpression. Representative images and the quantification of time for indicated processes in the control and D89E-expressing extruding cells. Asterisks indicate extruding cells. Crosses in a box-and-whisker plot indicate outliers.

(C) Quantification of the apical protrusion in control and D89E-expressing extruding MDCKII cells. Time 0 is defined as the start of cell extrusion (cell deformation).

(D) Immunostaining for cleaved caspase-3 with DAPI. Representative images of cleaved caspase-3-positive cells (asterisks) with protrusion (protrusion + ) or within the cell layer (protrusion -) and the distribution of cleaved caspase-3-positive cells with or without protrusion

15 in the control and D89E-expressing extruding cells. White or yellow dashed lines indicate the corresponding position in another angled view (x-y or x-z images).

(E) Fragmentation of membrane GFP-expressing extruding EpH4 cells. Representative images and the corresponding schematic views. Fragments (arrowheads) were not engulfed but remained in the intercellular space. Asterisks indicate extruding cells.

20 (F and G) Similar abnormalities in D89E-expressing EpH4 cells and D89E-expressing MDCKII cells: fragmentation and the quantification of time for indicated processes $(\mathrm{F})$ and apical protrusion (G) in control or D89E-expressing EpH4 cells, same as (B) and (C).

(H, I, and J) Phenotypes of Xkr8-siRNA EpH4 cells: PS exposure shown via Annexin V staining 
$(\mathrm{H})$, fragmentation shown via staining with CellMask and the quantification of time for indicated processes (I), and apical protrusion (J). Arrowheads indicate budding or shed vesicles. 


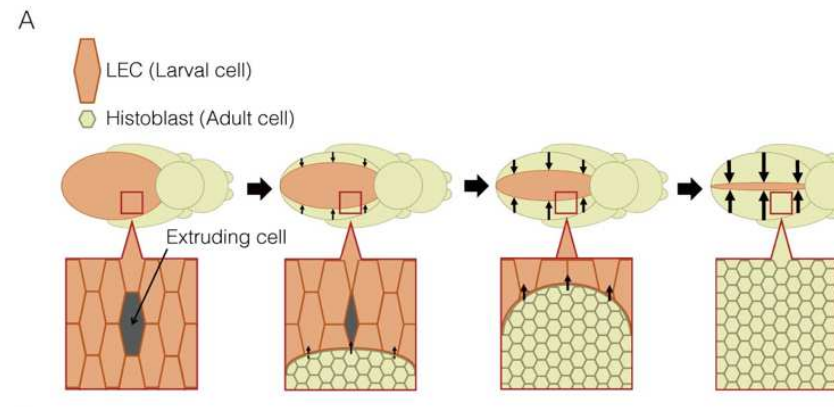

B
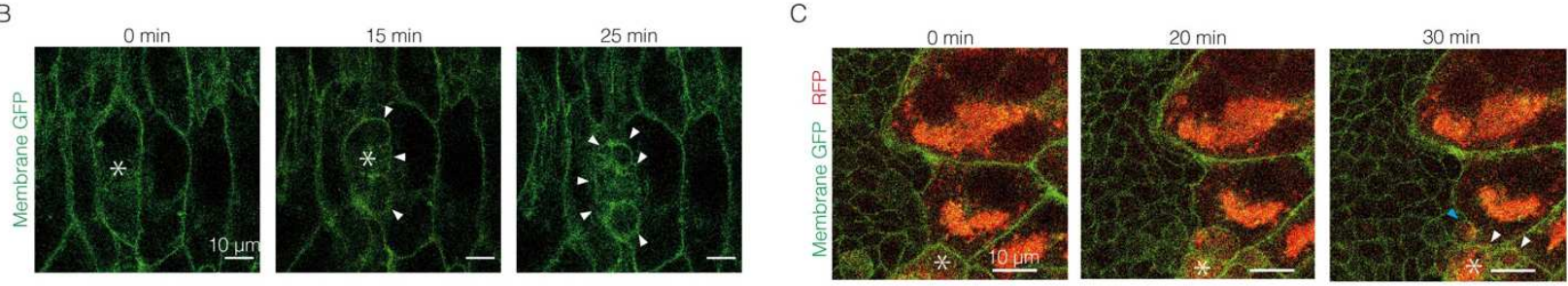

D
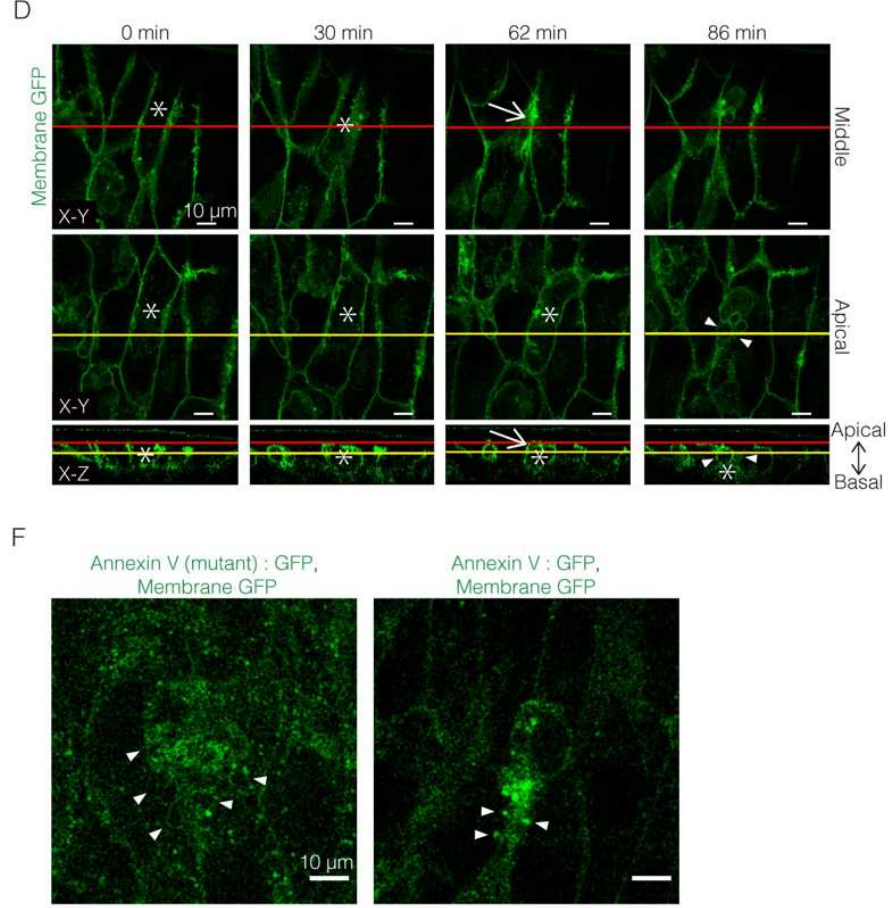

Annexin V : GFP

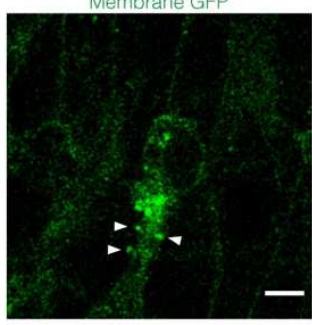

G

Annexin V (mutant) : GFP

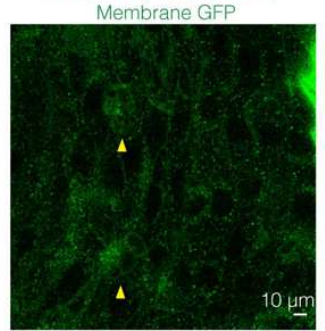

Annexin V: GFP, Membrane GFP

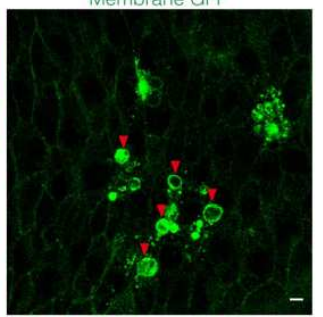

E
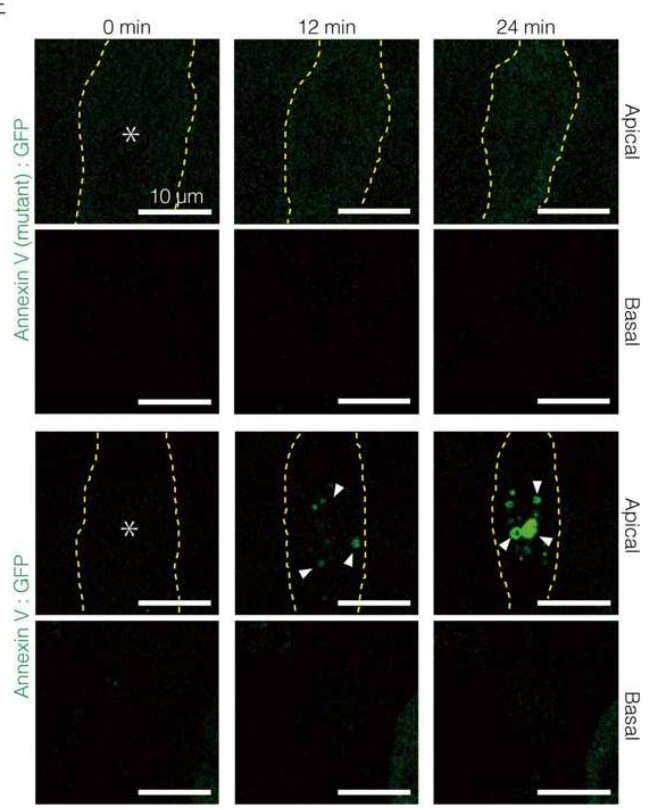

$\mathrm{H}$

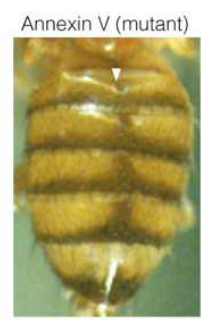

Annexin V

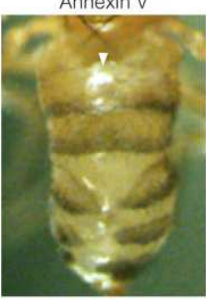



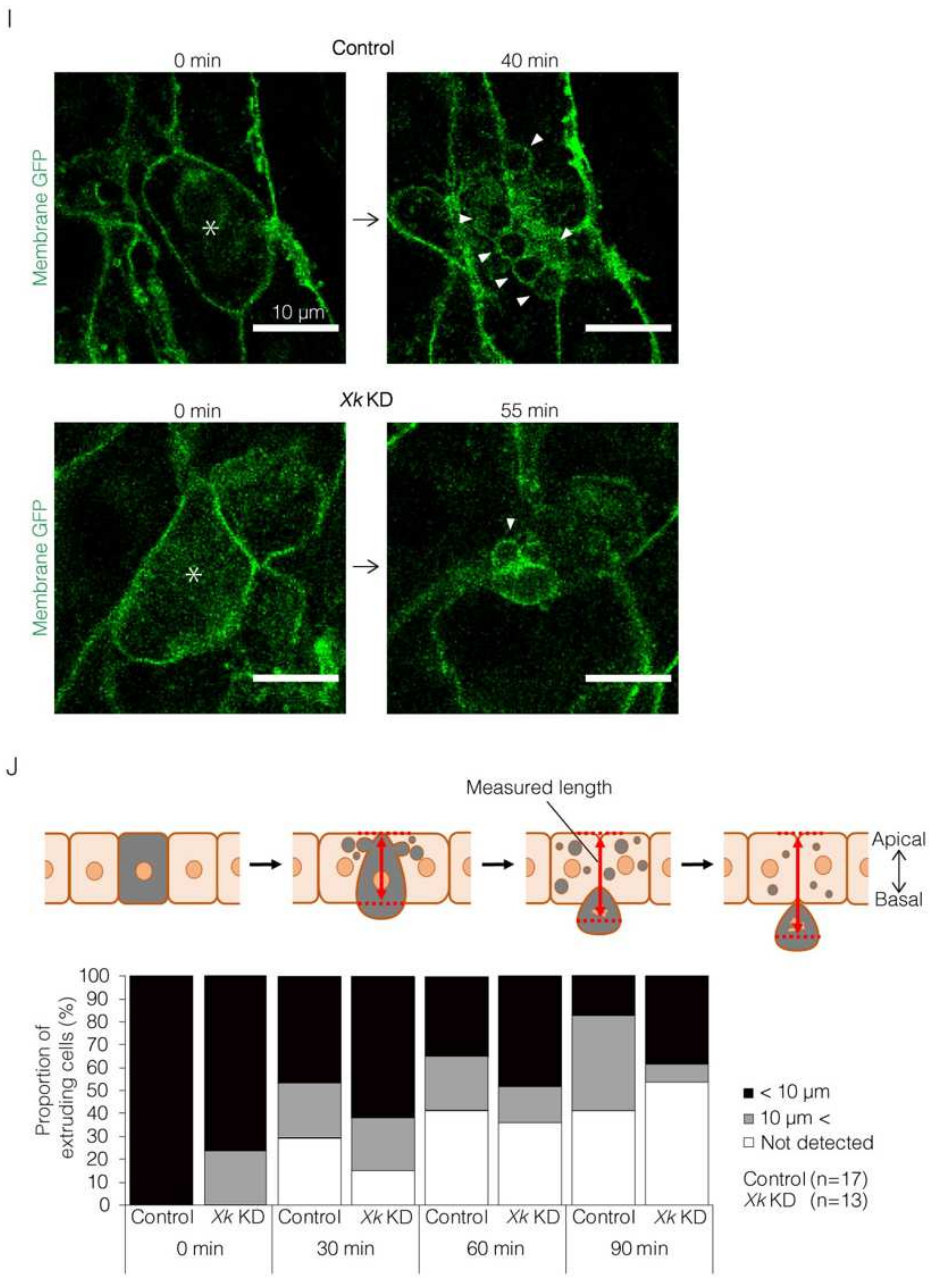

K
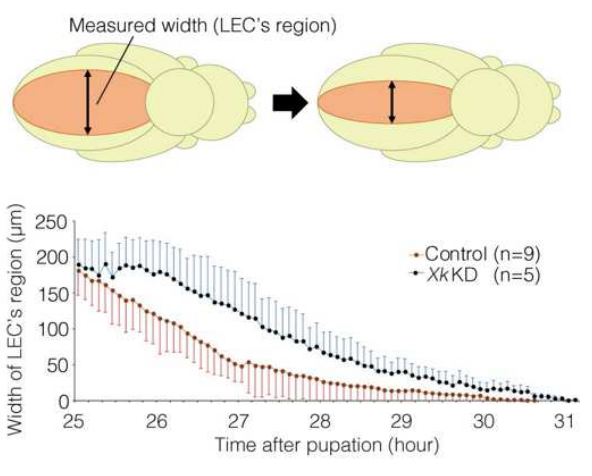

Fig. 3I-K 
Figure 3. PS exposure-mediated fragmentation of extruding cells promotes the execution of cell extrusion in Drosophila pupal epidermis to ensure tissue development

(A) Diagram of cell extrusion during the replacement of LECs with histoblasts in Drosophila pupal abdominal epidermis.

(B) Fragmentation (arrowheads) of an extruding LEC (asterisks) in Lgl:GFP (membrane GFP)expressing pupal epidermis.

(C) Pupal epidermis, in which only LECs express RFP and both LECs and histoblasts express Lgl:GFP (membrane GFP). White and blue arrowheads indicate extruding cell-derived RFPpositive vesicles in LECs and histoblasts, respectively. Asterisks indicate an extruding LEC. (D) Fragmentation (arrowheads) in Lgl:GFP-expressing extruding LEC (asterisks) occurred immediately after the apical surface closure (arrow) with neighboring cells. Note that fragmentation occurred at the apicolateral parts of LEC, which extrude to the basal direction. Apical and middle x-y sections with x-z sections are shown.

(E) Visualization of local PS exposure in Annexin V:GFP- or Annexin V mutant:GFP-expressing 15 extruding LECs (dotted lines).

(F) Less and smaller fragmentation in Annexin V:GFP- and Lgl:GFP- expressing extruding LEC compared with that in Annexin V nutant:GFP- and Lgl:GFP- expressing extruding LEC. Arrowheads indicate shed fragments.

(G) More remained extruding LECs (red arrowheads) in Annexin V:GFP- and Lgl:GFPexpressing epithelial cell layer of compared to that in Annexin V nutant:GFP- and Lgl:GFPexpressing epithelial cell layer. Yellow arrowheads indicate normally extruding cells with normal fragmentation.

(H) Dorsal abdomen of adult Drosophila expressing Annexin V:GFP or Annexin V mutant:GFP. 
Arrowheads indicate the midlines.

(I) Less fragmentation in Xk-RNAi LECs. Representative images of control or Xk-RNAi Lgl:GFP-expressing LEC. Arrowheads indicate shed fragments.

(J) Quantification of the progression of cell extrusion after the sealing of the apical surface by the neighboring cells. Diagram shows that the distance from the apical surface of cell layer to a section in which the width of the extruding LEC is at the maximum (red double-sided arrows). The distance is classified as either more or less than $10 \mu \mathrm{m}$ and its distribution at the indicated times and genotypes is shown in the graph. Not detected indicates either progressed or completed extrusion.

10 (K) Reduction in the width of the region covered by LECs (LEC's region) with time evolution in the control and Xk-RNAi pupal epidermis. Diagram shows the measured width. 
A
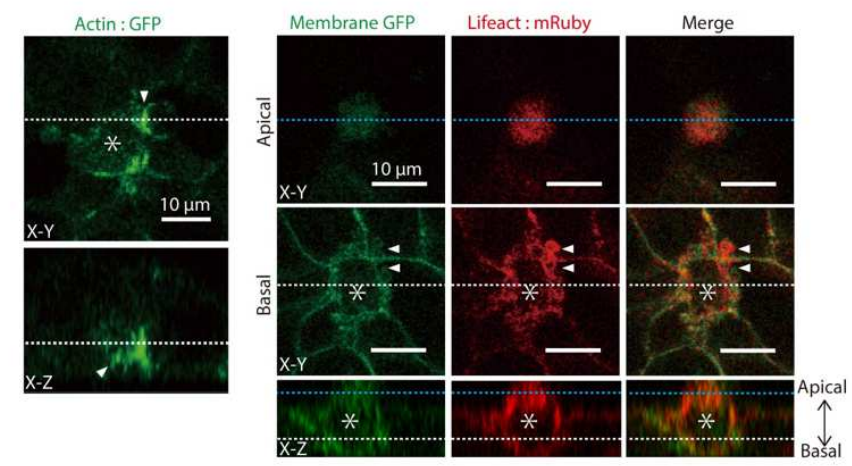

B

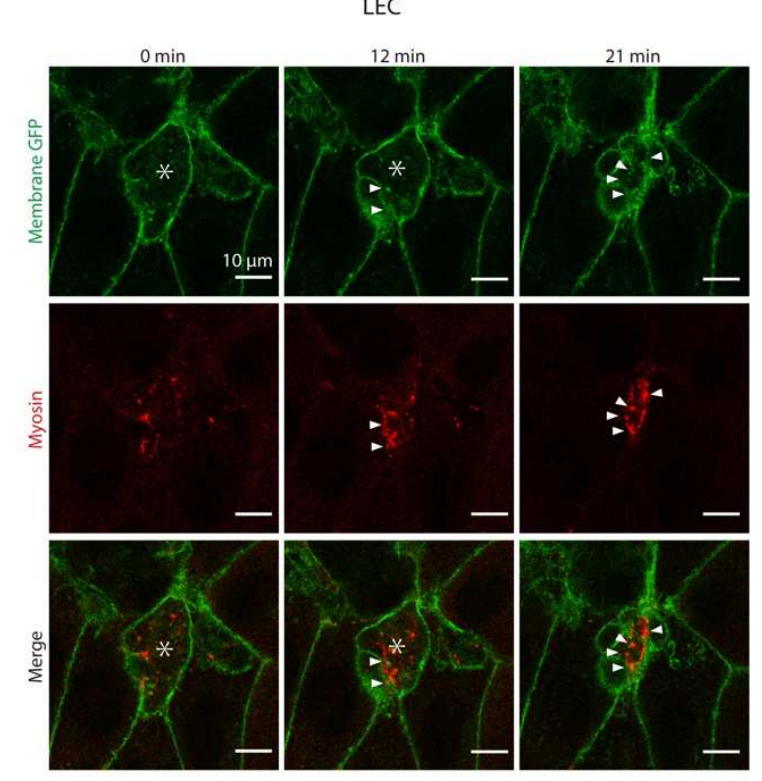

C

$\mathrm{EpH} 4$
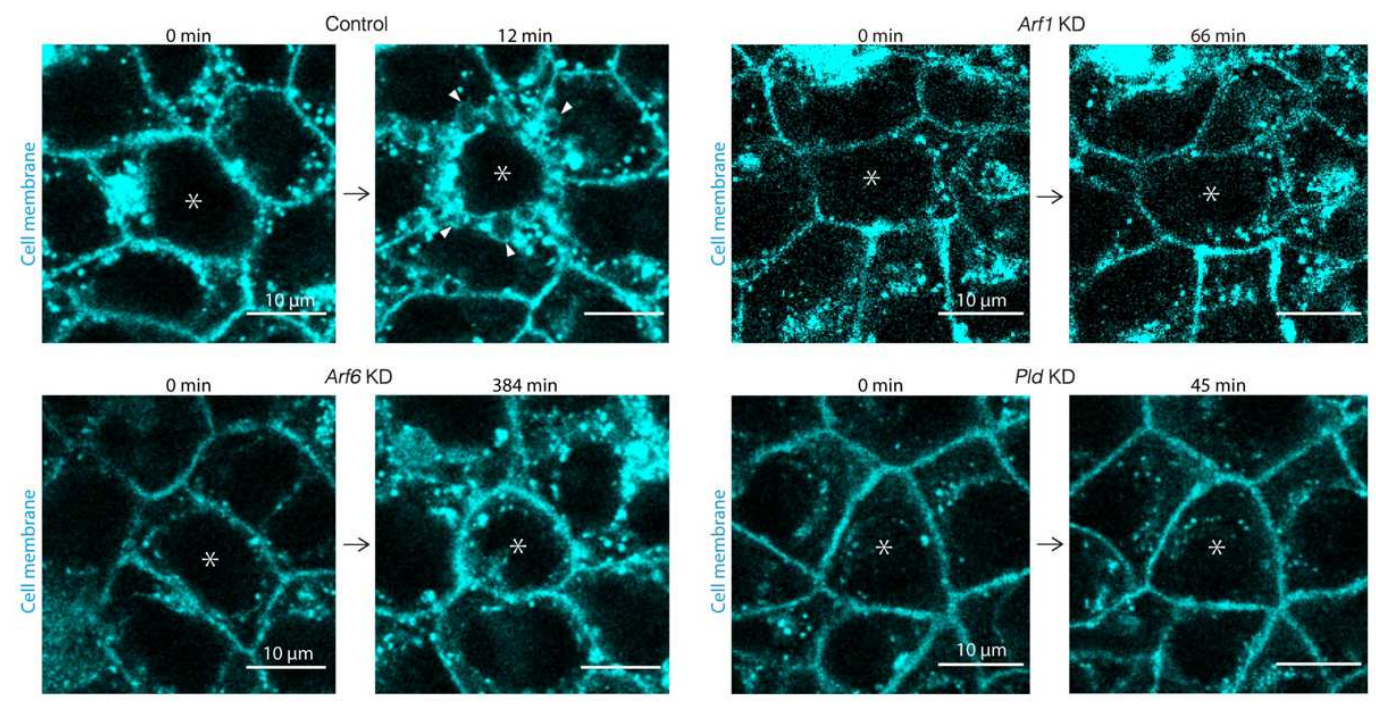

Fig. 4A-C 
C
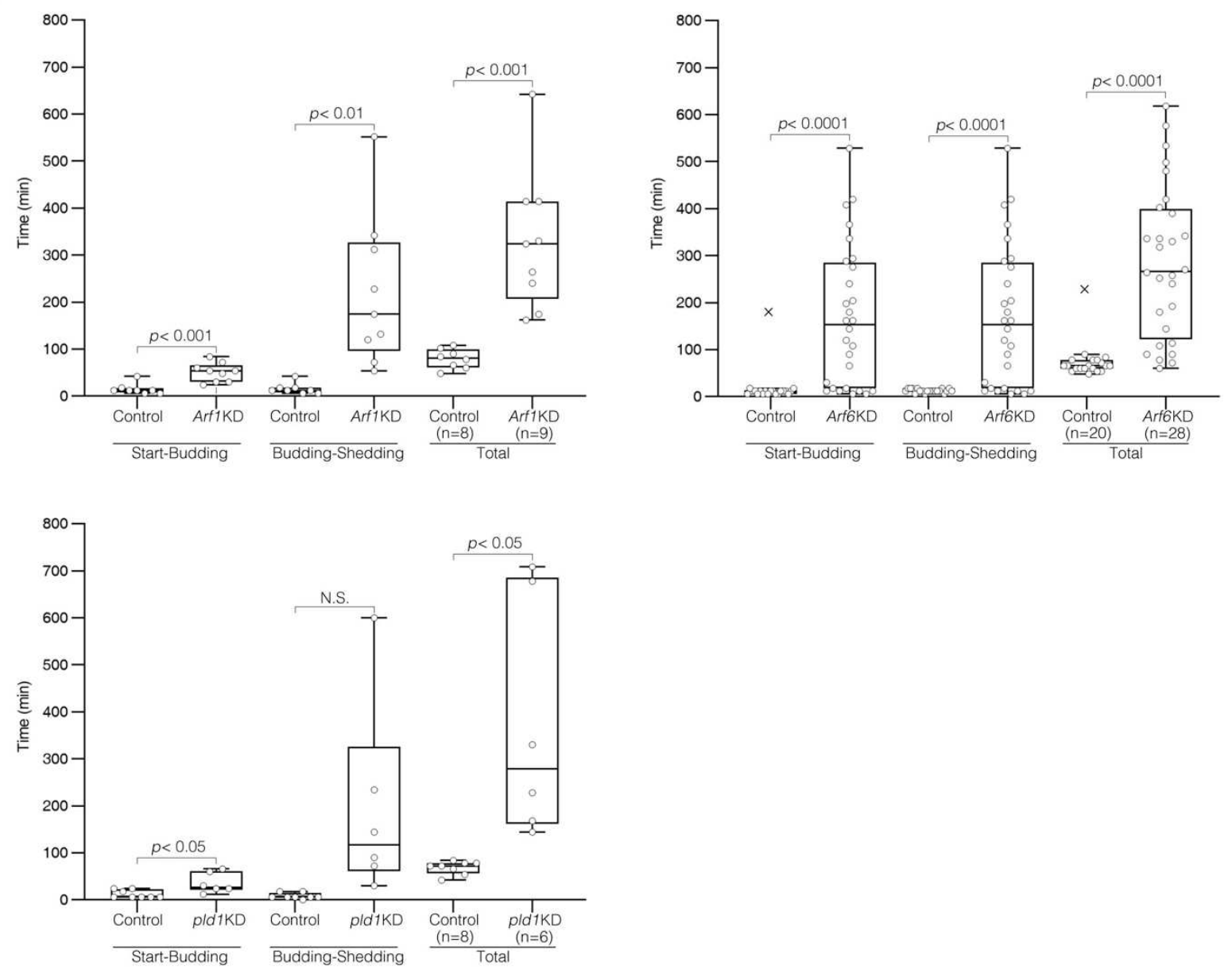

D
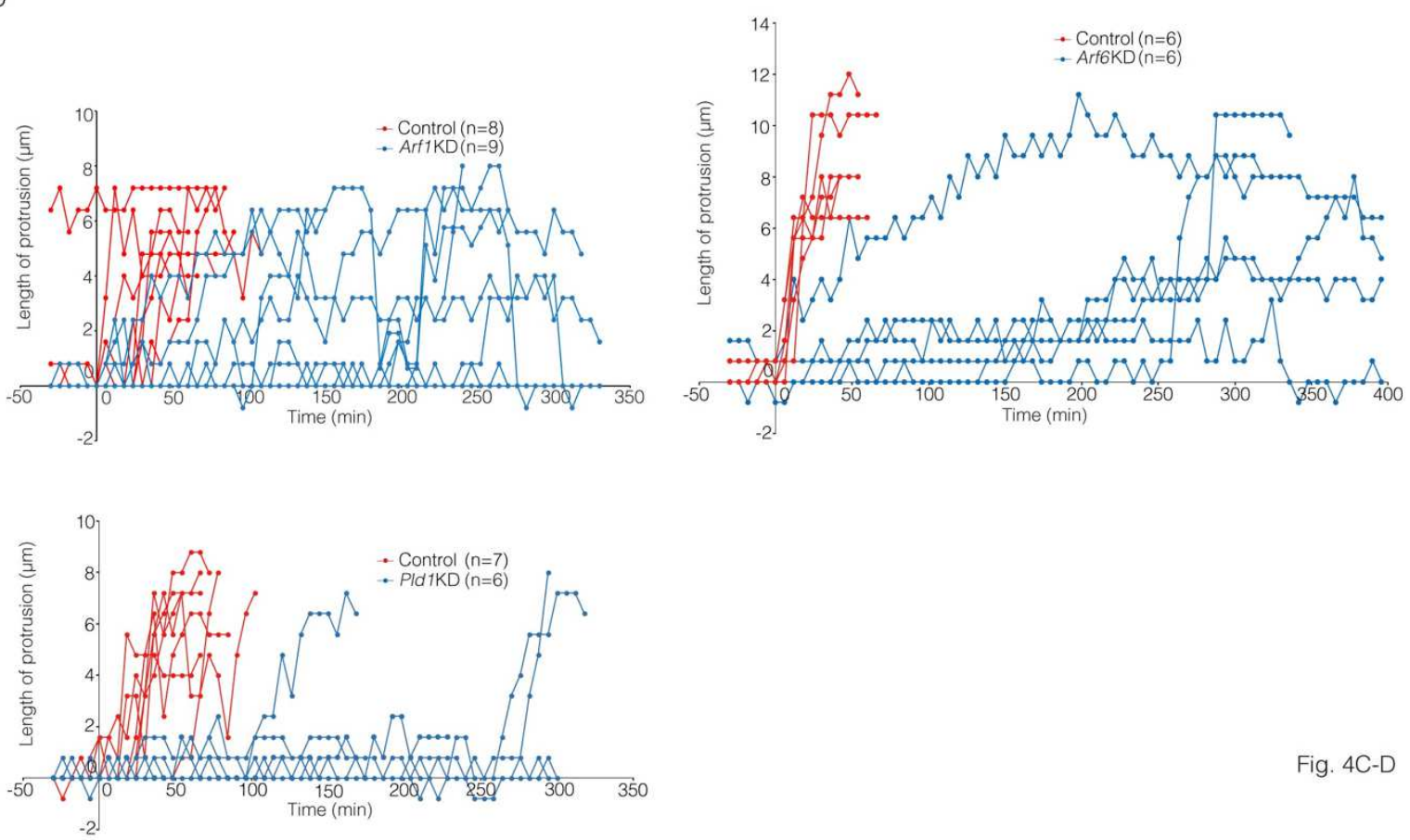

Fig. 4C-D 

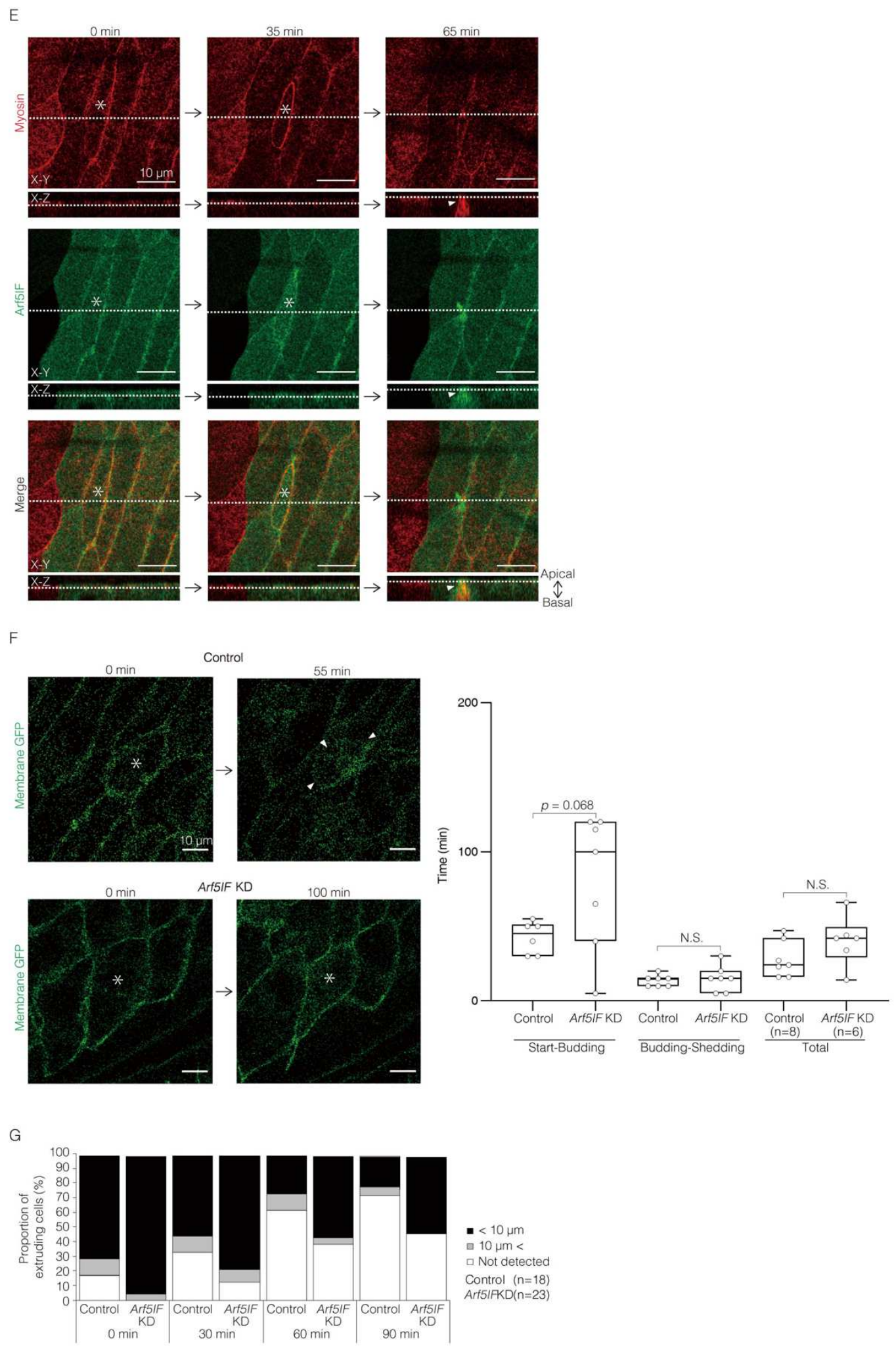

Fig. 4E-G 
$\mathrm{H}$
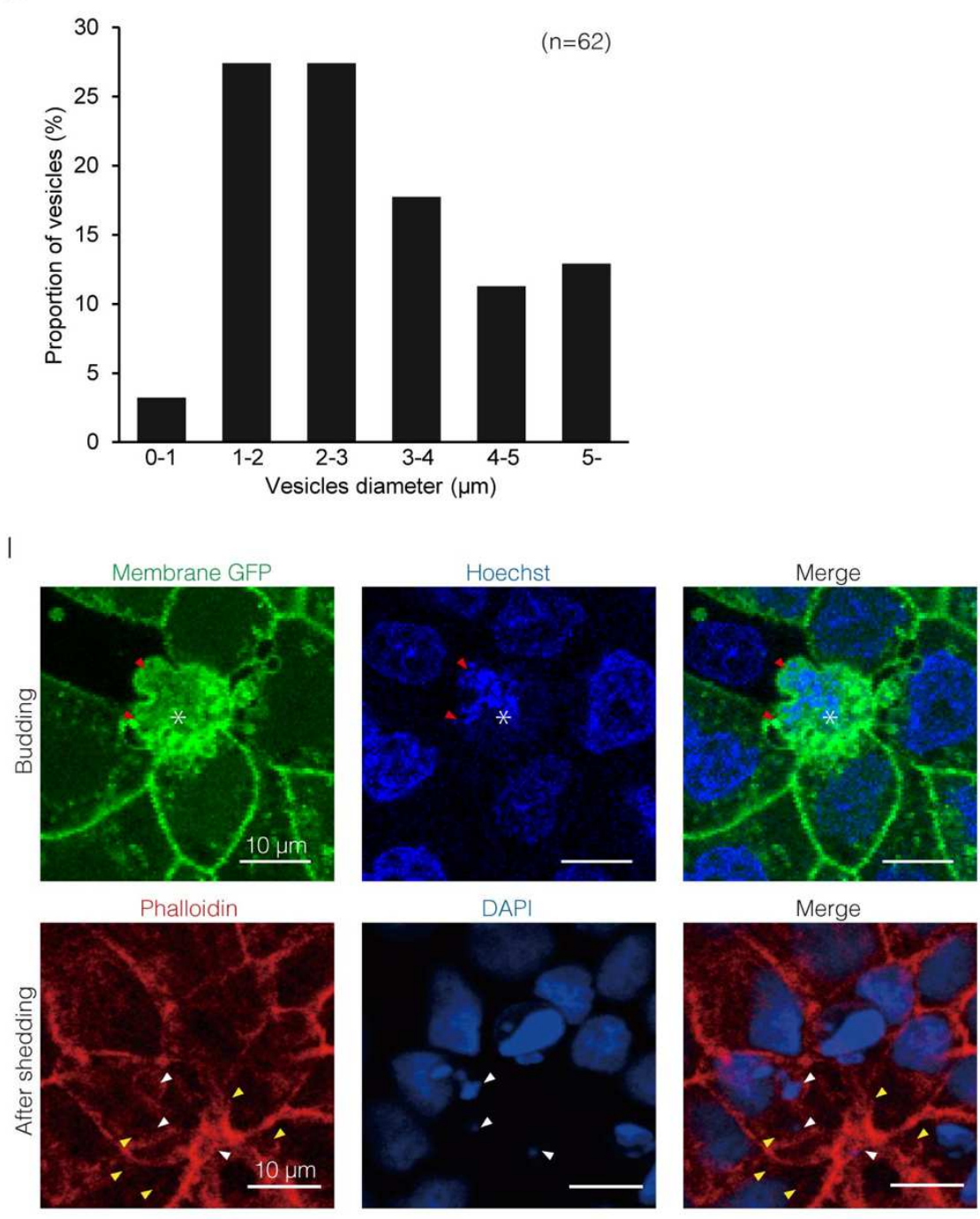

J

Before apoptosis

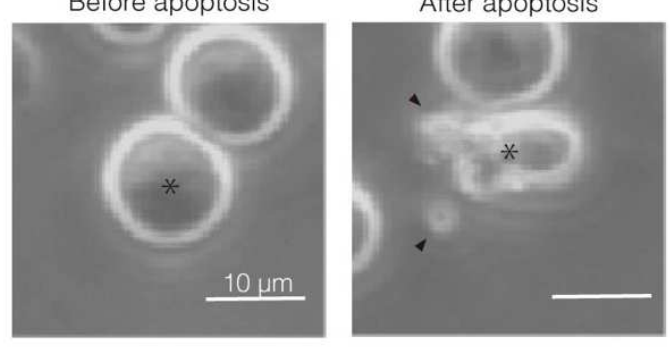

Control

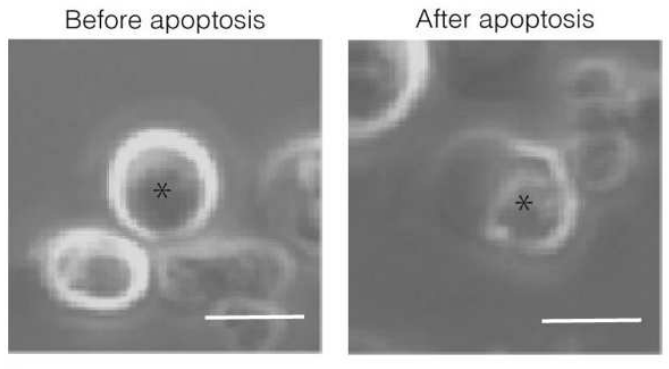

Pld1 inhibitor
Fig. $4 \mathrm{H}-\mathrm{J}$ 
Figure 4. Arf family- and PLD-mediated EV formation promotes efficient cell extrusion in mammalian cultured cells and Drosophila pupal epidermis

(A) Accumulation of actin (arrowheads) at the budding sites at the basal parts but not blebbing sites (rarely shed) of the apical surface in Actin:GFP-expressing and Lifeact:mRuby2- and membrane GFP-expressing extruding (asterisk) MDCKII cells.

(B) Accumulation of myosin (arrowheads) in Lgl:GFP- and sqh (myosin regulatory right chain):mKate2-expressing extruding LEC (asterisk).

(C) Representative images and the quantification of time for indicated processes in Arf6-, Arfl-, or Pld1- siRNA extruding Eph4 cells (asterisks) stained with CellMask. Arrowheads indicate

10 shed vesicles. Crosses in a box-and-whisker plot indicate outliers.

(D) Quantification of protrusion of the indicated EpH4 cells in Figure 4C. Time 0 is defined as the start of cell extrusion (cell deformation).

(E) Localization of Arf51F visualized in Arf51F:GFP- and sqh:mKate2-expressing pupal epidermis. Asterisks indicate an extruding LEC.

15 (F) Representative images and the quantification of time for indicated processes in the control and Arf5 1F-RNAi Lgl:GFP-expressing LECs. Arrowheads indicate shed vesicles.

(G) Distribution of extruding LECs for the indicated times in the control and Arf51F-RNAi pupal epidermis, same as Figure 3J.

(H) Distribution of shed vesicles produced by extruding EpH4 cells. (I) DNA in vesicles derived from extruding cells. Representative image of DNA in the vesicles (arrowheads) during budding (upper, red arrowheads) or after shedding and engulfment (lower, white arrowheads) of an extruding MDCKII cell (asterisks). DNA was stained with Hoechst (upper) or DAPI (lower) as well as the visualization of cell membrane and vesicles by membrane 
GFP (upper) or phalloidin (lower). Yellow arrowheads indicate the shed and engulfed vesicles without DNA.

(J) Representative bright field images of dying FDC-P1 cells without or with PLD1 inhibitor (VU0155069). Asterisks indicate dying cells and arrowheads indicate vesicles (apoptotic body). 
A

MDCK I I
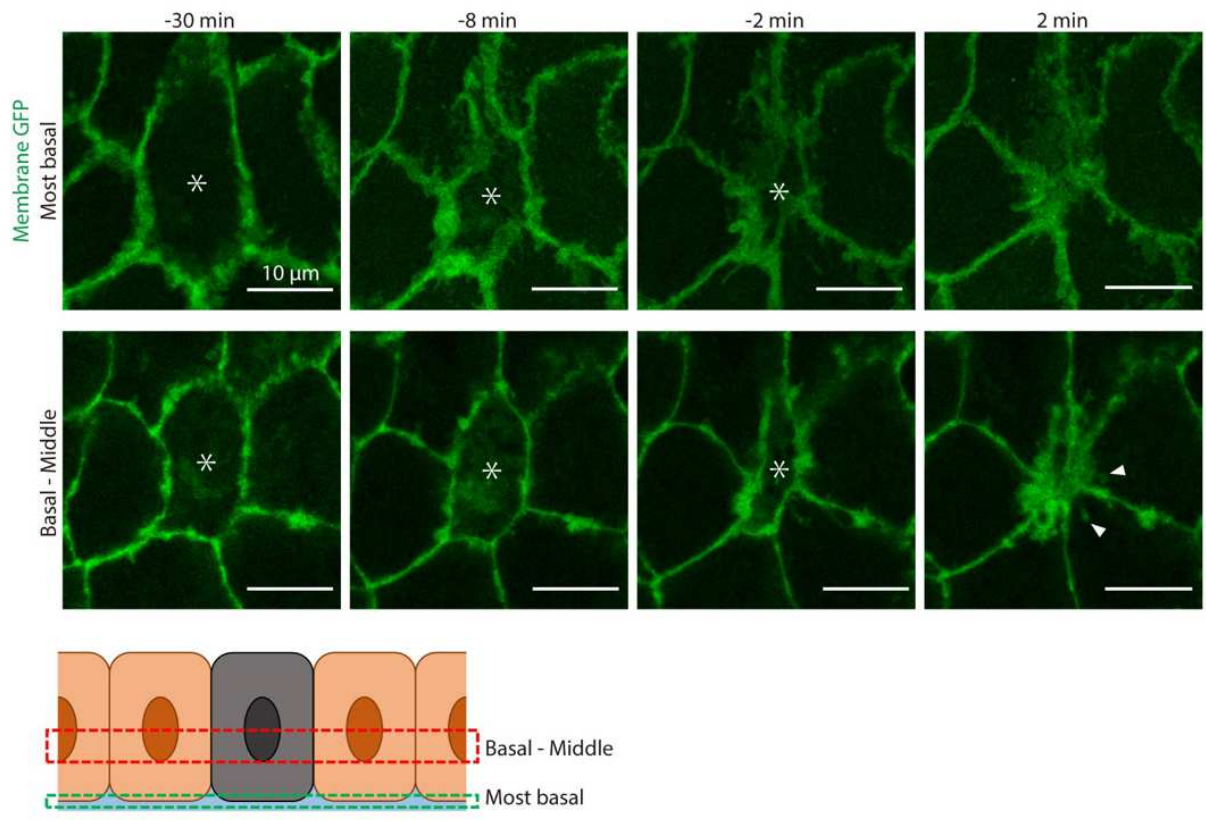

B

MDCK II (Most basal)
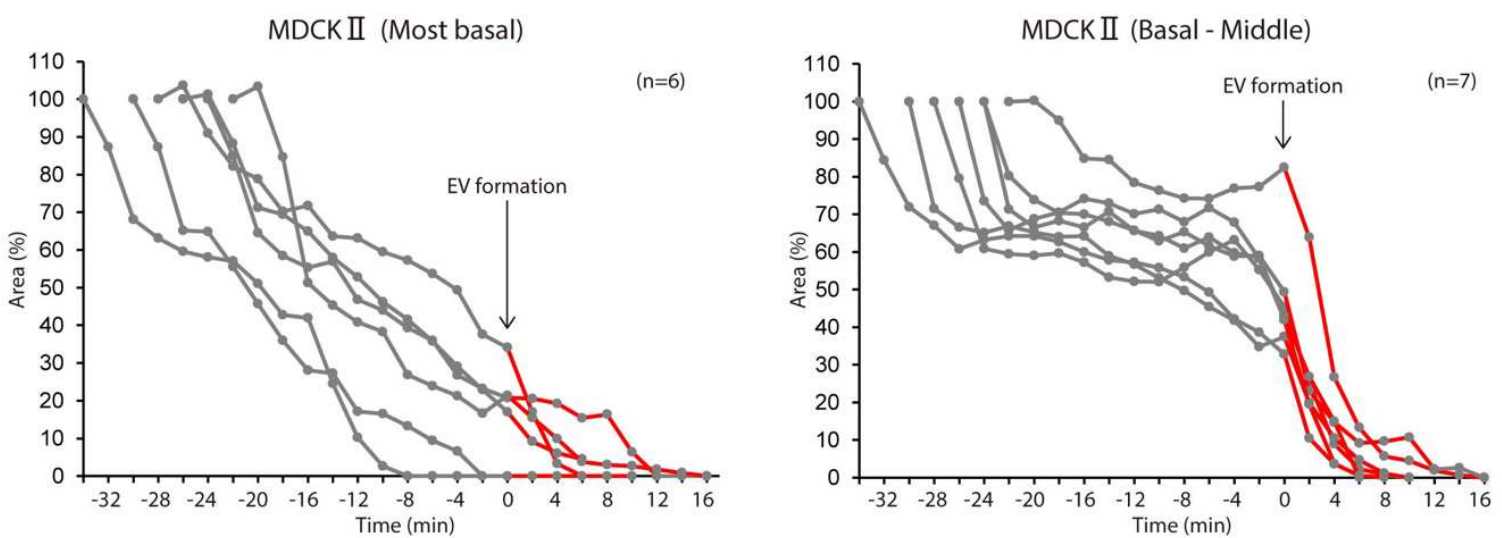

C

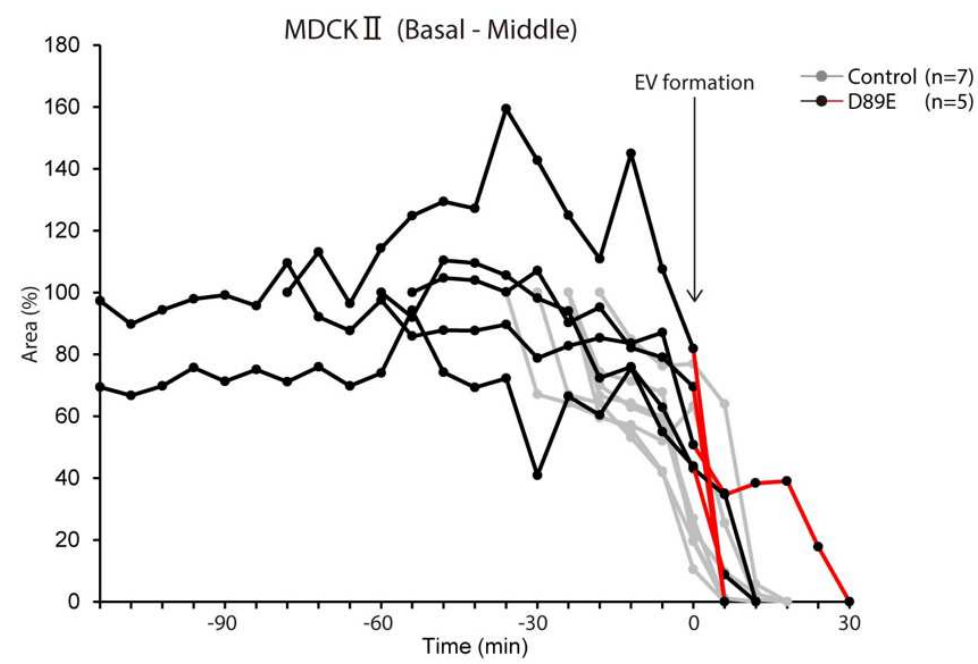

Fig. 5A-C 
D
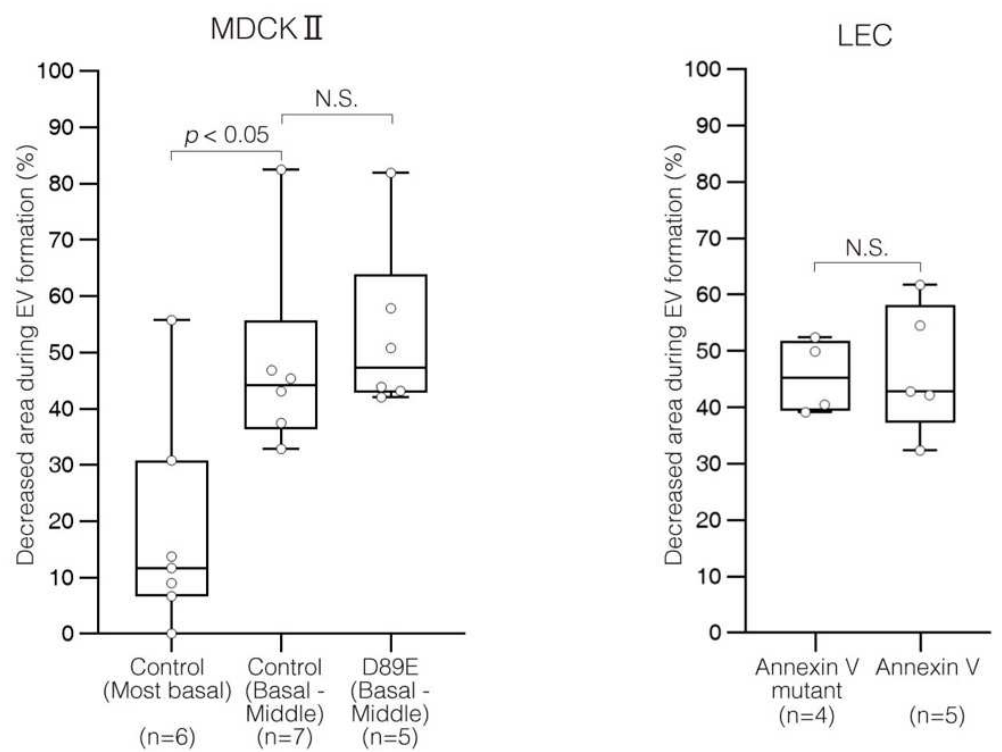

E

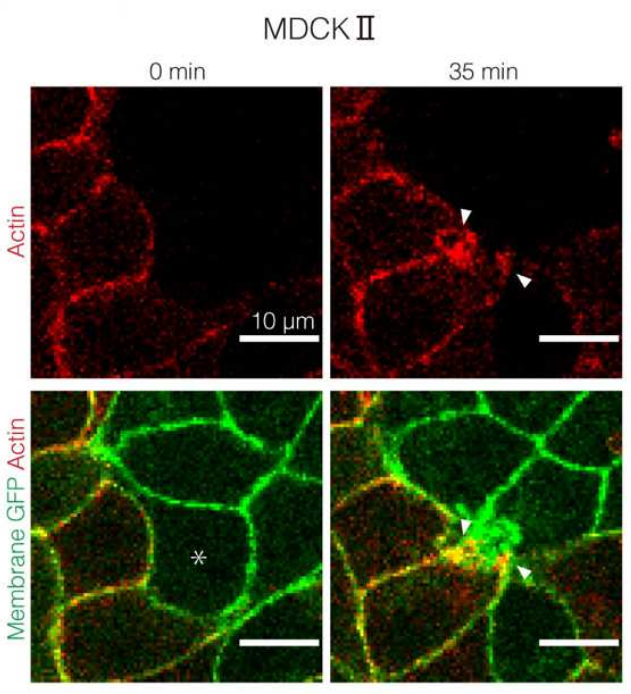

F

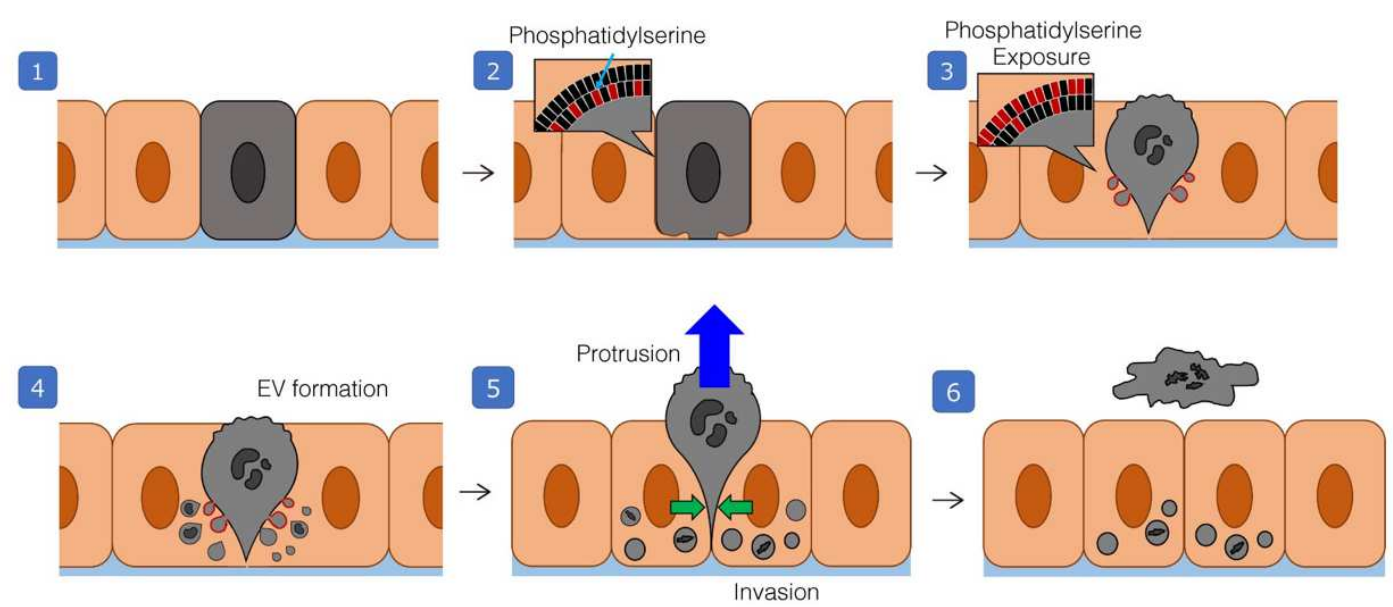

Fig. 5D-F 
Figure 5. EV formation propels cell extrusion by creating a space for neighboring cells invasion

(A) Representative images of membrane GFP-expressing extruding MDCKII cell (asterisks) at the $\mathrm{x}-\mathrm{y}$ planes at the most basal (Most basal) and the $\mathrm{x}-\mathrm{y}$ planes where EV formation most frequently occurs (Basal-Middle: basal half but closed to the border of apical and basal half). Arrowheads indicate shed EVs. Diagram of sagittal view shows the imaged planes. (B) Quantified evolution of cell area at the most basal and Basal-Middle x-y planes (shown in A) in membrane GFP-expressing extruding MDCKII cells. $100 \%$ is defined as the area at the start of cell extrusion. Red lines indicate the period of EV shedding. Time 0 is defined as the start of EV shedding (EV formation).

(C) Quantified evolution of cell area at the Basal-Middle x-y planes in membrane GFPexpressing extruding MDCKII cells with (black lines) or without (gray lines) MFG-E8 D89E overexpression, same as (B). Red lines indicate the period of EV shedding in MFG-E8 D89Eexpressing cells.

15 (D) Ratio of decreased area during EV shedding (i.e., EV formation) at the most basal and BasalMiddle x-y planes compared to the cell area at the start of cell extrusion in extruding MDCKII cells with or without MFG-E8 D89E-overexpression and Drosophila LECs with Annex V:GFP or Annex V mutant:GFP-overexpression.

(E) Representative images of membrane GFP alone- expressing extruding MDCKII cells 20 (asterisk) and Lifeact:mRuby2- and membrane GFP- expressing MDCKII cells in mosaic culture. Actin was accumulated at the leading edge in the neighboring cell (arrowheads). (F) Proposed model for the execution of cell extrusion based on our results. 
A

$A^{\prime}$

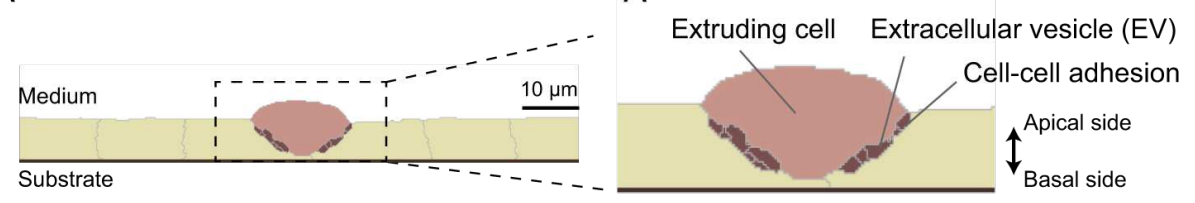

B

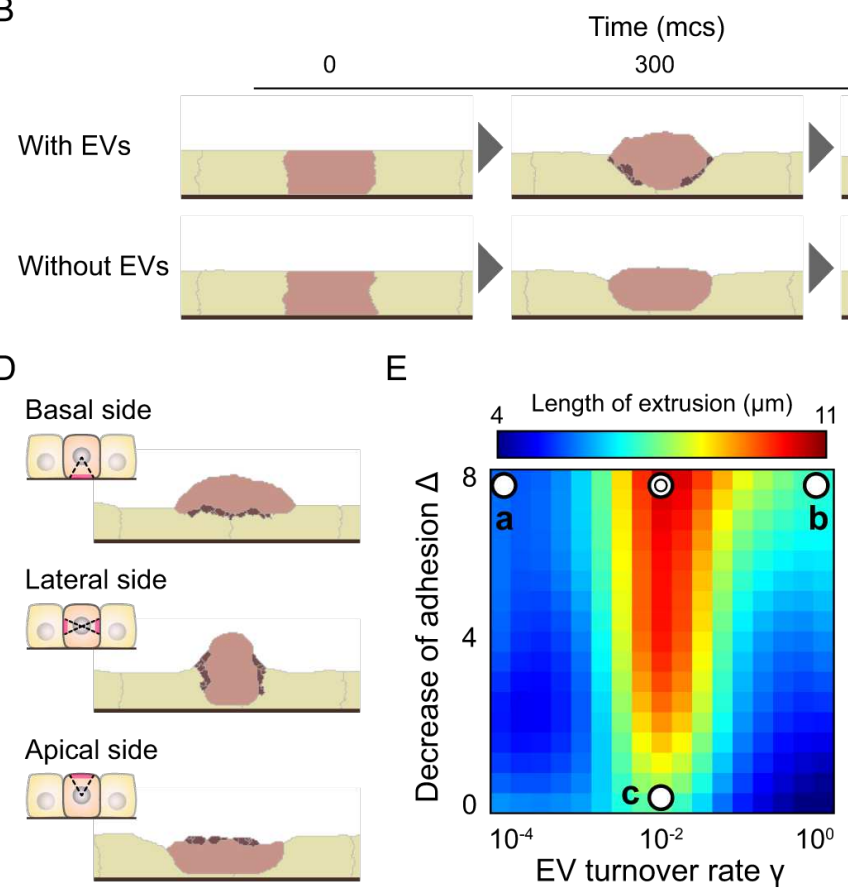

C

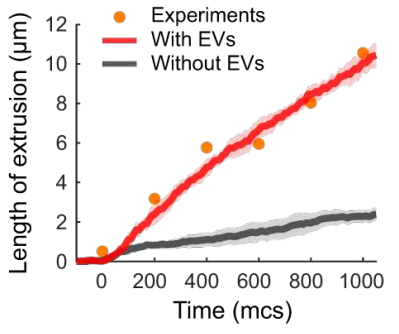

$\mathrm{F}$

Time (mcs)

$\begin{array}{lll}0 & 300 & 1000\end{array}$

a Slow EV turnover

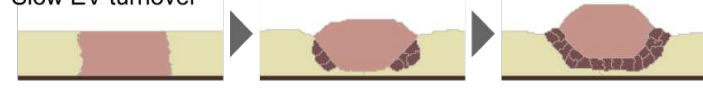

b Fast EV turnover

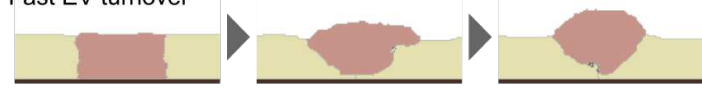

C No decrease in adhesion

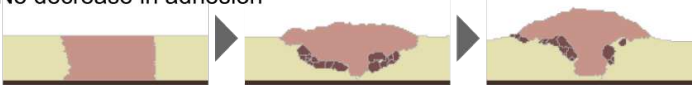

Figure 6 


\section{Figure 6. Model simulations show that EV formation promotes cell extrusion}

(A) Representative section view of cells vertical to the monolayer in the computational model. Scale bar, $10 \mu \mathrm{m}$. (A') Magnified view of (A) showing the extruding cell and EVs. The dynamics of the cells and EVs and the cell-cell adhesion are included in the model.

(B) Time course snapshots of morphological changes in the presence (upper) and absence (lower) of EVs. Time origin indicates the timing at the specification of the extruding cell. EV formation occurs at $\mathrm{mcs}=50$.

(C) Length of extrusion as a function of time with (red) and without (gray) EVs. The data represent the means \pm s.d. $(n=10)$. The orange dots represent the mean experimental data values $10 \quad(\mathrm{n}=5)$.

(D) Representative snapshots of cells and EVs in different regimes in terms of subcellular location of EV formation: EV formation on the basal/substrate side (upper), lateral side (middle), and apical/medium side (lower).

(E) Heatmap of the mean length of extrusion for EV turnover rate $(\gamma)$ and reduction of adhesion

$15(\Delta)$ at the final time step $(\operatorname{mcs}=1050) . \mathrm{n}=10$.

(F) Time course snapshots of the cells and EVs in different $\gamma$ and $\Delta$ : (a) slow EV turnover $\left(\gamma=10^{-}\right.$ $\left.{ }^{4}, \Delta=8\right)$, (b) fast EV turnover $\left(\gamma=10^{0}, \Delta=8\right)$, and (c) no reduction of adhesion $\left(\gamma=10^{-2}, \Delta=0\right)$. Small letters correspond to the parameter points in (E). 


\section{References}

Akers, J.C., Gonda, D., Kim, R., Carter, B.S., and Chen, C.C. (2013). Biogenesis of extracellular vesicles (EV): exosomes, microvesicles, retrovirus-like vesicles, and apoptotic bodies. J. Neurooncol. 113, 1-11.

5 Anton, K.A., Kajita, M., Narumi, R., Fujita, Y., and Tada, M. (2018). Src-transformed cells hijack mitosis to extrude from the epithelium. Nat Commun 9, 4695.

Atieh, Y., Wyatt, T., Zaske, A.M., and Eisenhoffer, G.T. (2021). Pulsatile contractions promote apoptotic cell extrusion in epithelial tissues. Curr Biol 31, 1129-1140.e4.

Catalano, M., and O'Driscoll, L. (2020). Inhibiting extracellular vesicles formation and release: a review of EV inhibitors. Journal of Extracellular Vesicles 9, 1703244.

Denning, D.P., Hatch, V., and Horvitz, H.R. (2012). Programmed elimination of cells by caspase-independent cell extrusion in C. elegans. Nature 488, 226-230.

Duszyc, K., Gomez, G.A., Lagendijk, A.K., Yau, M.-K., Nanavati, B.N., Gliddon, B.L., Hall, T.E., Verma, S., Hogan, B.M., Pitson, S.M., et al. (2021). Mechanotransduction activates RhoA in the neighbors of apoptotic epithelial cells to engage apical extrusion. Current Biology 31, 1326-1336.e5.

Dwivedi, V.K., Pardo-Pastor, C., Droste, R., Kong, J.N., Tucker, N., Denning, D.P., Rosenblatt, J., and Horvitz, H.R. (2021). Replication stress promotes cell elimination by extrusion. Nature 16.

20 Eisenhoffer, G.T., Loftus, P.D., Yoshigi, M., Otsuna, H., Chien, C.-B., Morcos, P.A., and Rosenblatt, J. (2012). Crowding induces live cell extrusion to maintain homeostatic cell numbers in epithelia. Nature 484, 546-549.

Fadul, J., Zulueta-Coarasa, T., Slattum, G.M., Redd, N.M., Jin, M.F., Redd, M.J., Daetwyler, S., Hedeen, D., Huisken, J., and Rosenblatt, J. (2021). KRas-transformed epithelia cells invade and partially dedifferentiate by basal cell extrusion. Nat Commun 12, 7180.

Gagliardi, P.A., Somale, D., Puliafito, A., Chiaverina, G., di Blasio, L., Oneto, M., Bianchini, P., Bussolino, F., and Primo, L. (2018). MRCK $\alpha$ is activated by caspase cleavage to assemble an apical actin ring for epithelial cell extrusion. J Cell Biol 217, 231-249.

Grieve, A.G., and Rabouille, C. (2014). Extracellular cleavage of E-cadherin promotes epithelial cell extrusion. J Cell Sci 127, 3331-3346.

Gu, Y., Shea, J., Slattum, G., Firpo, M.A., Alexander, M., Mulvihill, S.J., Golubovskaya, V.M., and Rosenblatt, J. (2015). Defective apical extrusion signaling contributes to aggressive tumor hallmarks. Elife 4, e04069.

Gudipaty, S.A., and Rosenblatt, J. (2017). Epithelial cell extrusion: Pathways and pathologies.

Semin. Cell Dev. Biol. 67, 132-140. 
Günther, C., Neumann, H., Neurath, M.F., and Becker, C. (2012). Apoptosis, necrosis and necroptosis: cell death regulation in the intestinal epithelium. Gut.

Gupta, K.H., Goldufsky, J.W., Wood, S.J., Tardi, N.J., Moorthy, G.S., Gilbert, D.Z., Zayas, J.P., Hahm, E., Altintas, M.M., Reiser, J., et al. (2017). Apoptosis and Compensatory Proliferation Signaling Are Coupled by CrkI-Containing Microvesicles. Dev. Cell 41, 674-684.e5.

Hanayama, R., Tanaka, M., Miwa, K., Shinohara, A., Iwamatsu, A., and Nagata, S. (2002). Identification of a factor that links apoptotic cells to phagocytes. Nature 417, 182-187.

Hoshika, S., Sun, X., Kuranaga, E., and Umetsu, D. (2020). Reduction of endocytic activity accelerates cell elimination during tissue remodeling of the Drosophila epidermal epithelium. Development 147.

Kocgozlu, L., Saw, T.B., Le, A.P., Yow, I., Shagirov, M., Wong, E., Mège, R.-M., Lim, C.T., Toyama, Y., and Ladoux, B. (2016). Epithelial cell packing induces distinct modes of cell extrusions. Curr Biol 26, 2942-2950.

Kuipers, D., Mehonic, A., Kajita, M., Peter, L., Fujita, Y., Duke, T., Charras, G., and Gale, J.E. (2014). Epithelial repair is a two-stage process driven first by dying cells and then by their neighbours. Journal of Cell Science 127, 1229-1241.

Le, A.P., Rupprecht, J.-F., Mège, R.-M., Toyama, Y., Lim, C.T., and Ladoux, B. (2021). Adhesion-mediated heterogeneous actin organization governs apoptotic cell extrusion. Nat Commun 12, 397.

Li, W., and Baker, N.E. (2007). Engulfment is required for cell competition. Cell 129, 12151225 .

Lubkov, V., and Bar-Sagi, D. (2014). E-cadherin-mediated cell coupling is required for apoptotic cell extrusion. Curr Biol 24, 868-874.

Marinari, E., Mehonic, A., Curran, S., Gale, J., Duke, T., and Baum, B. (2012). Live-cell delamination counterbalances epithelial growth to limit tissue overcrowding. Nature 484, $542-$ 545.

Michael, M., Meiring, J.C.M., Acharya, B.R., Matthews, D.R., Verma, S., Han, S.P., Hill, M.M., Parton, R.G., Gomez, G.A., and Yap, A.S. (2016). Coronin 1B Reorganizes the Architecture of F-Actin Networks for Contractility at Steady-State and Apoptotic Adherens Junctions. Dev. Cell 37, 58-71.

Michel, M., and Dahmann, C. (2020). Tissue mechanical properties modulate cell extrusion in the Drosophila abdominal epidermis. Development 147.

Muralidharan-Chari, V., Clancy, J., Plou, C., Romao, M., Chavrier, P., Raposo, G., and D'Souza-Schorey, C. (2009). ARF6-Regulated Shedding of Tumor Cell-Derived Plasma Membrane Microvesicles. Current Biology 19, 1875-1885. 
Nagata, S., Hanayama, R., and Kawane, K. (2010). Autoimmunity and the clearance of dead cells. Cell 140, 619-630.

Nakajima, Y., Kuranaga, E., Sugimura, K., Miyawaki, A., and Miura, M. (2011).

Nonautonomous Apoptosis Is Triggered by Local Cell Cycle Progression during Epithelial

Replacement in Drosophila. Mol. Cell. Biol. 31, 2499-2512.

Ohsawa, S., Sugimura, K., Takino, K., Xu, T., Miyawaki, A., and Igaki, T. (2011). Elimination of Oncogenic Neighbors by JNK-Mediated Engulfment in Drosophila. Developmental Cell 20, 315-328.

Ohsawa, S., Vaughen, J., and Igaki, T. (2018). Cell Extrusion: A Stress-Responsive Force for

Good or Evil in Epithelial Homeostasis. Dev. Cell 44, 284-296.

Resnik-Docampo, M., Koehler, C.L., Clark, R.I., Schinaman, J.M., Sauer, V., Wong, D.M., Lewis, S., D'Alterio, C., Walker, D.W., and Jones, D.L. (2017). Tricellular junctions regulate intestinal stem cell behaviour to maintain homeostasis. Nat Cell Biol 19, 52-59.

Sapar, M.L., Ji, H., Wang, B., Poe, A.R., Dubey, K., Ren, X., Ni, J.-Q., and Han, C. (2018).

Phosphatidylserine Externalization Results from and Causes Neurite Degeneration in Drosophila. Cell Reports 24, 2273-2286.

Schlienger, S., Campbell, S., and Claing, A. (2014). ARF1 regulates the Rho/MLC pathway to control EGF-dependent breast cancer cell invasion. Mol. Biol. Cell 25, 17-29.

Sun, Z., Amourda, C., Shagirov, M., Hara, Y., Saunders, T.E., and Toyama, Y. (2017).

Basolateral protrusion and apical contraction cooperatively drive Drosophila germ-band extension. Nat Cell Biol 19, 375-383.

Sung, B.H., Parent, C.A., and Weaver, A.M. (2021). Extracellular vesicles: Critical players during cell migration. Developmental Cell.

Suzuki, J., Denning, D.P., Imanishi, E., Horvitz, H.R., and Nagata, S. (2013). Xk-related protein 8 and CED-8 promote phosphatidylserine exposure in apoptotic cells. Science 341, 403-406.

Suzuki, J., Imanishi, E., and Nagata, S. (2014). Exposure of Phosphatidylserine by Xk-related Protein Family Members during Apoptosis. J. Biol. Chem. 289, 30257-30267.

Tanimura, N., and Fujita, Y. (2020). Epithelial defense against cancer (EDAC). Seminars in Cancer Biology 63, 44-48.

30 Teng, X., Qin, L., Le Borgne, R., and Toyama, Y. (2017). Remodeling of adhesion and modulation of mechanical tensile forces during apoptosis in Drosophila epithelium.

Development 144, 95-105.

Thomas, M., Ladoux, B., and Toyama, Y. (2020). Desmosomal Junctions Govern Tissue Integrity and Actomyosin Contractility in Apoptotic Cell Extrusion. Curr Biol 30, 682-690.e5. 
Tkach, M., and Théry, C. (2016). Communication by Extracellular Vesicles: Where We Are and Where We Need to Go. Cell 164, 1226-1232. 


\section{Supplementary Files}

This is a list of supplementary files associated with this preprint. Click to download.

- MovieS1.mp4

- Movies2.mp4

- MovieS3A.mp4

- MovieS3B.mp4

- MovieS4.mp4

- MovieS5.mp4

- MovieS6.mp4

- MovieS7A.mp4

- MovieS7B.mp4

- Movies8.mp4

- Movies9.mp4

- MovieS10.mp4

- Supplementarylnformation.pdf 